Review Article

\title{
Old and Recent Advances in Life Cycle, Pathogenesis, Diagnosis, Prevention, and Treatment of Malaria Including Perspectives in Ethiopia
}

\author{
Dejen Nureye $\mathbb{i}^{1}$ and Solomon Assefa $\mathbb{i D}^{2}$ \\ ${ }^{1}$ Department of Pharmacy, College of Medicine and Health Sciences, Mizan-Tepi University, \\ Mizan Campus, P.O. Box 260, Southwest, Mizan-Aman, Ethiopia \\ ${ }^{2}$ Department of Pharmacology and Clinical Pharmacy, College of Health Sciences, School of Pharmacy, Addis Ababa University, \\ P.O. Box 1176, Addis Ababa, Ethiopia
}

Correspondence should be addressed to Dejen Nureye; dejenureye@gmail.com

Received 2 July 2019; Accepted 16 January 2020; Published 14 February 2020

Academic Editor: Adriano Sfriso

Copyright (c) 2020 Dejen Nureye and Solomon Assefa. This is an open access article distributed under the Creative Commons Attribution License, which permits unrestricted use, distribution, and reproduction in any medium, provided the original work is properly cited.

\begin{abstract}
Malaria, caused by apicomplexan parasite, is an old disease and continues to be a major public health threat in many countries. This article aims to present different aspects of malaria including causes, pathogenesis, prevention, and treatment in an articulate and comprehensive manner. Six Plasmodium species are recognized as the etiology of human malaria, of which Plasmodium falciparum is popular in East and Southern Africa. Malaria is transmitted mainly through Anopheles gambiae and Anopheles funestus, the two most effective malaria vectors in the world. Half of the world's population is at risk for malaria infection. Globally, the morbidity and mortality rates of malaria have become decreased even though few reports in Ethiopia showed high prevalence of malaria. The malaria parasite has a complex life cycle that takes place both inside the mosquito and human beings. Generally, diagnosis of malaria is classified into clinical and parasitological diagnoses. Lack of clear understanding on the overall biology of Plasmodium has created a challenge in an effort to develop new drugs, vaccines, and preventive methods against malaria. However, three types of vaccines and a lot of novel compounds are under perclinical and clinical studies that are triggered by the occurrence of resistance among commonly used drugs and insecticides. Antiadhesion adjunctive therapies are also under investigation in the laboratory. In addition to previously known targets for diagnostic tool, vaccine and drug discovery scientists from all corner of the world are in search of new targets and chemical entities.
\end{abstract}

\section{Introduction}

The term malaria was derived from the Italian word "mala aria" meaning foul air [1]. It is a protozoal blood infection caused by a mosquito-borne apicomplexan parasite, which is transmitted to humans during the bite of an infected female Anopheles mosquito species [2,3]. The United States National Institute of Allergy and Infectious Diseases (NIAID) defines malaria as a disease caused by a parasite that lives part of its life in humans and part in mosquitoes [4]. This review aims to present all aspects of malaria in a coherent and comprehensive manner. An attempt was made to give introductory concepts regarding history, causative agents, prevalence, and incidence of malaria. It also provides old and new notions about the cell biology, pathophysiology, diagnosis, and management of malaria in one umbrella including some tips from Ethiopia. In advance, we seek to summarize recent developments in drug, vaccine, and control measures of malaria.

Malaria is an ancient disease that could be traced back to the very earliest human history. It was accepted as a disease by Hippocrates in the fourth century BC [5]. In the early seventeenth Century, the Peruvian bark of Cinchona tree was known to treat fever [6]. In 1847, Heinrich Meckel identified black-brown pigment granules in the blood and spleen of an insane person [7]. Othmer Zeidler synthesized 
Dichloro-Diphenyl-Trichloroethane (DDT) in 1874 for his thesis. Alphonse Laveran noticed parasites, which he called Oscillaria malariae, in the blood of a malaria patient in 1880 [8]. The genus plasmodium was portrayed by Ettore Marchiafava and Angelo Celli in 1885 [9]. The whole transmission cycle of the parasite was elucidated in 1897 by Ronald Ross. In 1898, Camillo Golgi and others demonstrated that human malaria was transmitted by Anopheles mosquitoes [10]. Chloroquine was discovered in 1934 by Hans Andersag. He called his compound resochin. In early 1950s, malaria was thought to be eliminated from the USA. Then after, human infection with Plasmodium knowlesi was recognized in 1965. Artemisinin was isolated from the plant Artemisia annua in 1971 [6, 10]. Next, a polymerase chain reaction- (PCR-) based malaria detection was depicted in the early 1990s, and meanwhile malaria rapid diagnostic tests (RDTs) were developed [11].

The genus Plasmodium (the causative agent for malaria) is thought to have originated from Dinoflagellates (photosynthetic protozoa). From more than 200 different species of Plasmodium, at least 13 species are pathogenic to humans [9]. Five of them, falciparum, vivax, ovale (two species), and malariae, are well-known etiology of human malaria. Moreover, disease with knowlesi occur in people when an Anopheles mosquito infected by a monkey bites humans [12].

Of these species, falciparum (dominant in East and Southern Africa) is mainly prevalent on the African continent and is responsible for most deaths from malaria. Plasmodium vivax has a wider geographic distribution. Although it can occur throughout Africa, the risk of infection with vivax is quite low there because of the absence of Duffy gene in many African populations [13]. There is, however, a growing evidence that vivax is being transmitted among Duffy blood group-negative inhabitants in Africa including Ethiopia [14].

Malaria is transmitted majorly through bites of the genus Anopheles mosquitoes, which includes more than 537 recognized species [15]. The two most efficient malaria vectors in the world, A. gambiae and A. funestus, are primary malaria vectors in Africa [16]. A. gambiae, A. funestus, and A. pharoensis were confirmed as the principal vectors in Ethiopia [14]. Due to residence of the parasite in RBCs, malaria can also be transmitted through blood transfusion, organ transplant, or shared use of needles or syringes contaminated with blood. A new-born baby may acquire congenital malaria before or during delivery [17]. Furthermore, malaria transmission is largely affected by global weather patterns, including El Nino and La Nina [18].

Around $44 \%$ of the world's population is at risk from malaria [19]. According to the latest estimates, 219,000,000 malaria cases, most (92\%) from World Health Organization (WHO) African Region, occurred globally in 2017, and the disease led to 435,000 deaths, most (93\%) of which were also in WHO African Region. Almost all (99.7\%) cases due to malaria are resulted from falciparum. In 2017, the number of global malaria mortality in children less than five years is estimated to be 266,000 [20]. Pregnant women have increased susceptibility to falciparum malaria. In malaria- endemic areas, falciparum contributes to $8-14 \%$ of low birth weight, which in turn decreases the chance of a baby's survival [6].

Global malaria case incidence was reduced by $59 \%$ in 2017. A reduction in mortality rates $(44.1 \%)$ was also reported in this year [20]. Not only health related impact but also there is a severe economic burden in terms of lost days of work due to the disease. Of course, malaria is considered to take off $1.3 \%$ from the economic growth and $40 \%$ of public health expenditure of some African countries. It affects developing countries in many aspects including determent of tourism [21].

Malaria was one of the top ten as well as major infectious diseases in Ethiopia [22, 23]. About 28,548,422 people of Ethiopia live in the high risk area for malaria infection. In 2017, Ethiopian Federal Ministry of Health (FMoH) reported 1,530,739 confirmed malaria cases and 356 deaths [20]. Despite decreased malaria occurrence rate and death rate in Ethiopia since 2010 [20], high prevalence was observed in some areas in contrast to high household coverage of control interventions $[24,25]$. This increment may be associated with individuals having poor socioeconomic status [26]. Ethiopia has achieved only half of the millennium development reduction target of malaria. For this reason, the country must strengthen its malaria control and treatment approaches to attain the sustainable development goals [27].

\section{Life Cycle of Malaria Parasite}

The human malaria parasite has a complex life cycle as shown in Figure 1. The motile infectious form, Plasmodium sporozoite, is passed to individuals when the insect bites the skin, probes for a blood vessel from which to feed, releases various vasodilators to increase its chance of finding a vessel and salivate into the blood to prevent clotting. Within 30-60 min of inoculation, the thread-like sporozoites are carried to the liver by the circulatory system $[4,5]$.

Over a period of 7-12 days, the sporozoites grow into schizonts and can develop up to 30,000 merozoites, which rupture the hepatocytes $[28,29]$. On the other hand, some vivax and ovale sporozoites turn into hypnozoites, a form that can remain latent in the liver for months or years and cause relapses in infected people [30]. Interestingly, recurrence of falciparum malaria was reported in patients some years after leaving an endemic area. It tells that, at least occasionally, falciparum has a dormant stage [31-33]. Then, the asexual cycle begins (Figure 1), with the merozoites invading $\mathrm{RBC}$ to grow by consuming hemoglobin. Within the host RBC, the parasite undergoes development from the early ring stage to late trophozoite and then after mitotic divisions to the schizont stage, which contains 6 to 32 merozoites, depending on the parasite species [34].

When the erythrocytic schizont ruptures, the released merozoites continue the life cycle by invading other RBCs. Cyclical fevers are typically happening shortly before or at the time of RBC lysis as schizonts rupture to release new infectious merozoites. This occurs every $48 \mathrm{~h}$ in tertian malaria and every $72 \mathrm{~h}$ in quartan malaria infection. During 


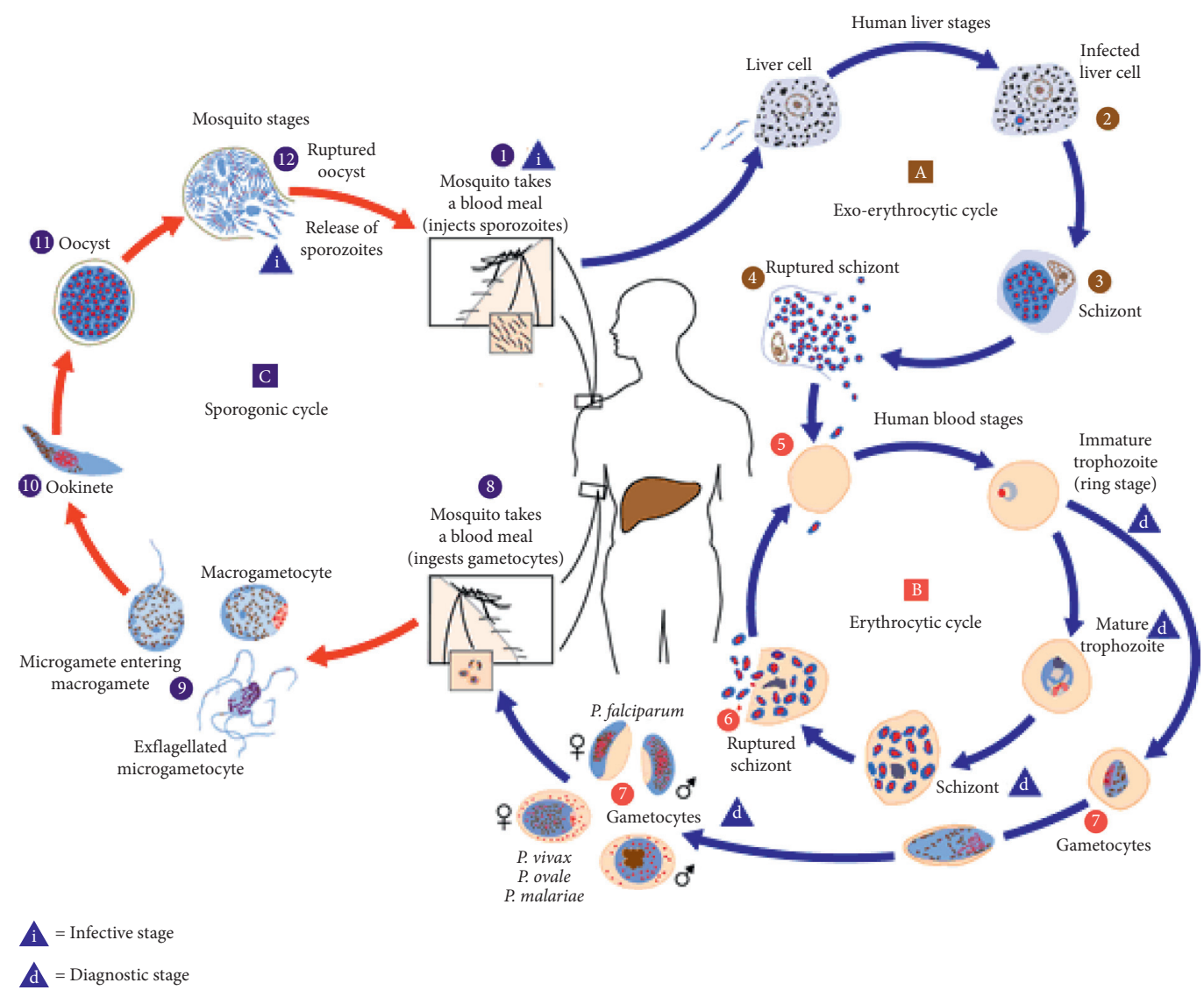

Figure 1: Life cycle of malaria parasites (adopted from [35]).

this repeated cycle, some merozoites differentiate into male and female sexual forms known as erythrocytic gametocytes with one nucleus and then awaiting the arrival of a bloodseeking female Anopheles mosquito [4, 34].

Then intake of gametocytes by the mosquito induces gametogenesis. The flagellated forms of microgametes, formed by exflagellation, penetrate or fertilize the macrogametes generating zygotes. The zygotes change into ookinetes and then become a round oocyst. Inside the oocyst, the nucleus divides repeatedly, with the formation of a large number of sporozoites and enlargement of the oocyst [28]. When the sporozoites are fully formed, the oocyst bursts, releasing the sporozoites into the haemocoel (the mosquito's body cavity). The sporozoites migrate to the salivary glands, thus completing the life cycle (Figure 1). Entrance of the sporozoites from the mosquito's salivary glands into a new human host perpetuates the malaria life cycle $[6,28]$.

\section{Cell Biology and Pathogenesis of Malaria}

The cell biology of Plasmodium is suggested to be similar to other eukaryotes since members of this genus are eukaryotic microbes. However, the whole biology of the parasite and its pathogenesis is not clearly known at the molecular and cellular levels [36, 37]. Here, we compiled old and recent facts to inform and click experts to involve them in developing vaccines, novel drugs, and malaria control tools, since somehow in-depth understanding on the biology of malaria parasite is critical to engage into these activities. All Apicomplexa including malaria parasites are characterized by a set of apical organelles called rhoptries, dense granules, and micronemes. In Plasmodium spp., there are three invasive forms: sporozoite, merozoite, and ookinete involving the apical organelles localized at one end of the parasite [38].

First, the sporozoite injected into the host skin enters the blood stream and quickly accesses the liver by a process called traversal. Proteins necessary for traversal are reviewed in [39]. The coreceptors on sporozoites (e.g., thrombospondin (TSP) domains on the circumsporozoite protein (CSP)) that mediate invasion [40] bind specifically to glycoaminoglycan chains of the heparan sulfate proteoglycans (HSP) on hepatocytes and Kupffer cells [41]. At least, two receptors, CD81and CD68, are found to be responsible for falciparum entry and invasion in hepatocytes. After penetrating space of Disse in the liver, sporozoites migrate through several hepatocytes and engage in a final invasion, with the formation of a parasitophorous vacuolar membrane (PVM). PVM is then ruptured by plasmodial proteolytic enzymes and merozoite egress from the infected hepatocyte to access blood circulation $[42,43]$.

Within the circulation, merozoite surface protein-1 (MSP-1) associated with the parasite membrane through a glycosylphosphatidylinositol (GPI) anchor does binds to the RBC surface proteins [44]. Eight other merozoite surfacebound GPI-anchored proteins interacting with RBC have been reviewed elsewhere [45]. Recently, CD55 (a protein on 
the surface of RBCs) was identified that serves as an essential gateway point for malaria parasite into RBCs. This discovery opens up a new path for the development of therapies to treat and prevent malaria [46]. After binding to RBC, the merozoite reorients itself using apical membrane antigen 1 (AMA-1) so that the apical end of the parasite will locate adjacent to the RBC membrane with a transient RBC deformation. The contents of apical organelles are going to be expelled as the parasite invades $[47,48]$.

Following reorientation and microneme discharge, a junction was formed between the parasite and host cell using microneme proteins that recognize and bind to receptors in the host $[37,49]$. Proteins in the neck of rhoptry such as RON2 are inserted into the host membrane and bind to AMA-1 to form the tight junction [50]. Then, the contact area becomes free of RBC membrane proteins. After this, a merozoite enzyme (serine protease) results in a localized disruption of the submembrane cytoskeleton and lipid architecture of RBC [51]. Formation of the junction triggers the release of rhoptry bulb, providing proteins and lipids required for the parasitophorous vacuole [52]. So, an incipient PVM will be formed in the junction area [53]. The junction between the parasite and host becomes like a ring and the parasite appears to move via this annulus as it enters the expanding parasitophorous vacuole [54]. Following doorway, the PVM and host cell membrane become closed [55].

The invasive forms of apicomplexan parasites are motile forms that crawl along the substratum by "gliding motility" (Figure 2). During invasion, the parasite literally crawls into the host cell via the moving junction. In gliding motility, the micronemes must be continuously released as the organism is moving and continuous formations of new junctions occur between the zoite and substratum. A myosin unique to the Apicomplexa would be anchored into the inner membrane complex (IMC) lying under the plasma membrane. The IMC-associated myosin will be interacted with actin as part of the glidesome. The various adhesins making up the moving junction (MJ) complex are then linked to the glidesome [55].

The myosin propels the actin filaments toward the posterior of the zoite. As myosin is anchored into the IMC, it does not move. Therefore, the transmembrane adhesins are pulled via the fluid lipid bilayer of the plasma membrane. Thus, the complex of adhesins and actin filaments is transported towards the posterior of the cell to produce forward motion of the parasite. When the adhesins reach the posterior end of the parasite, they are proteolyitcally cleaved and shed from the zoite surface and a trail of adhesive molecules is left behind the moving zoite on the substratum $[37,56]$.

Once inside the RBC, the parasite modifies the host cell to make it a more suitable environment. Formation of knobs, cytoadherence, and rosetting (Figure 3) are the major host cell changes which happen in the pathogenesis of malaria. Knob-associated histidine-rich protein (KAHRP) and erythrocyte membrane protein-2 (PfEMP2) are two of the several proteins which reorganize the host RBC submembrane cytoskeleton and induce knob formation [57]. A polymorphic protein, PfEMP1, has been anchored to the knobs by KAHRP and has become exposed on the host RBC surface and functions as a ligand. Other cytoadherence ligands [58] are shown in the right side of Figure 3.

The red cells infected with mature forms of the parasites adhere to the capillary and postcapillary venular endothelium in the deep microvasculature which leads to sequestration of the parasites in various organs such as the heart, lung, brain, liver, kidney, intestines, adipose tissue, and placenta. This feature of the disease has been related exclusively to falciparum $[59,60]$. However, it has also been seen in reticulocytes infected with vivax [61]. To adhere to the endothelium, PfEMP1 appears on the surface of the infected red blood cells (IRBCs) about $16 \mathrm{~h}$ after the invasion [62]. This antigenic variant molecule can bind to several adhesion receptors, as shown in the left side of Figure 3, expressed on the endothelial cells. Among these receptors, intercellular adhesion molecule-1 (ICAM-1) is a major sequestration receptor and serves as a rolling receptor $[63,64]$.

Sequestration is also seen during pregnancy when IRBC binds to placental chondroitin sulfate A (CSA), which is mediated by Variant Two chondrotin Sulphate A Antigen (VAR2CSA) [65]. So, placental malaria can cause miscarriage, intrauterine growth retardation, low birth weight, and congenital malaria [66]. Parasites sequestration provides them the microaerophilic venous environment that is better suited for their maturation, and adhesion allows them to escape clearance by the spleen and to hide from the immune system. IRBCs also adhere to uninfected RBCs to form red cell rosetting and to other parasitized RBCs to form agglutination [67].

In rosette formation, PfEMP1 has been shown to bind to complement receptor-1 (CR-1), heparin sulfate (HS), and ABO blood group [68, 69]. The lectin-like duffy-binding domain (DBL) of PfEMP1 can make strong adhesion with carbohydrate structures, particularly blood group A antigen [70]. That is why non O-blood groups are risk factors for lifethreatening malaria through enhanced rosette formation $[69,71]$. Falciparum, vivax, and ovale are all able to form rosettes $[72,73]$, but only those caused by falciparum have been associated with severe malaria [74]. Vivax and ovale show a marked penchant for young RBCs, while malariae prefers old cells. As a result, these parasites have low parasitemia level in the bloodstream. Plasmodium falciparum, however, can invade RBCs of all ages and produces very high parasitemia level $[46,75]$.

If the above-mentioned pathophysiologic process goes on uninhibited, it ultimately blocks blood flow, limits local oxygen supply, impedes mitochondrial ATP synthesis, and stimulates cytokine production, all these factors contributing to the development of a severe disease [76]. Additionally, the host RBC ruptures or lyses to enable parasite egress as subtilisin-like protease 1- (SUB1-) processed MSP-1 interacts with the spectrin network of the RBC cytoskeleton [77]. Along with lysis of IRBC, toxins (red cell membrane products, hemozoin pigment, and GPI) are also released into the blood and activate macrophages and endothelial cells to secrete cytokines and inflammatory mediators. The systemic 


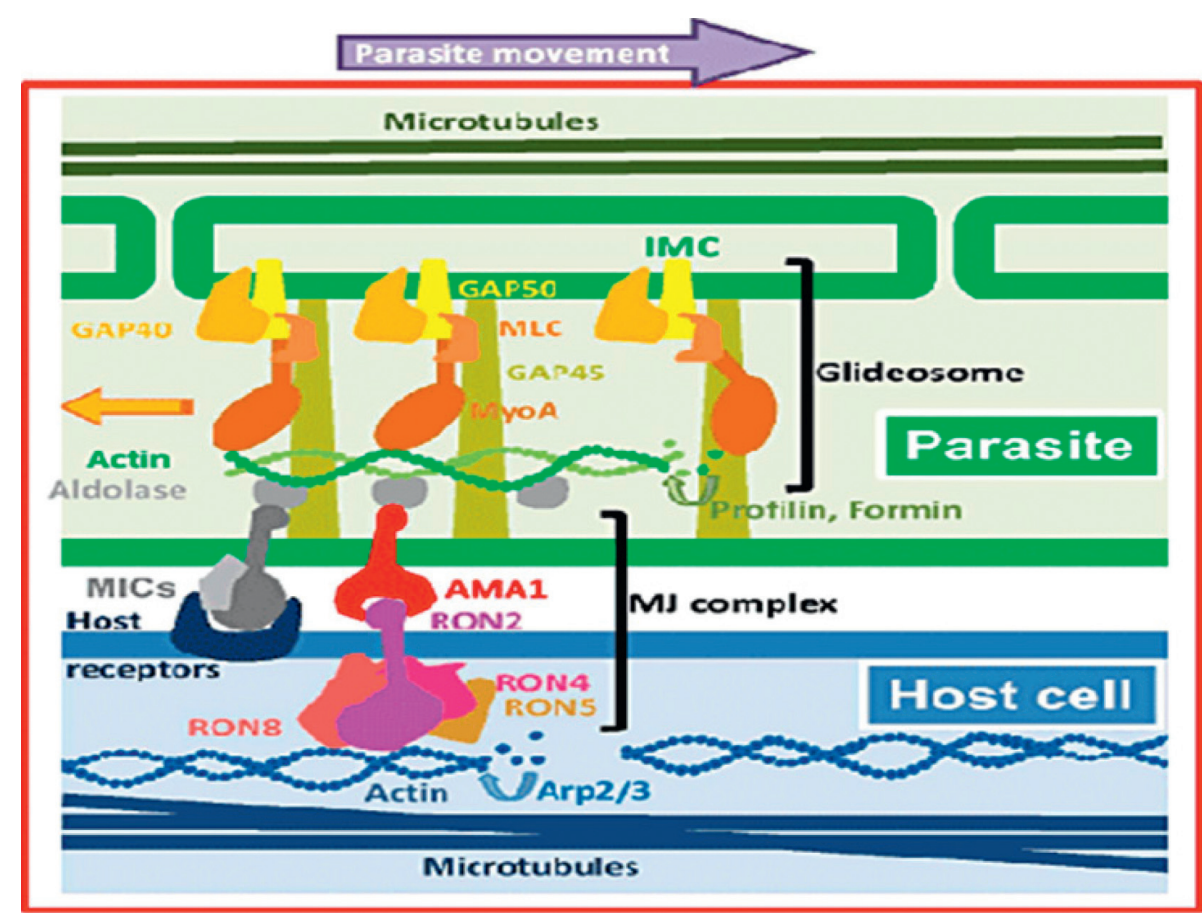

FIGURE 2: Model of the moving junction complex and glidesome driving gliding motility (GAP: gliding-associated protein, MyoA: myosin A MLC: myosin light chain, and Arp2/3: actin nucleator complex) (adopted from [55]).

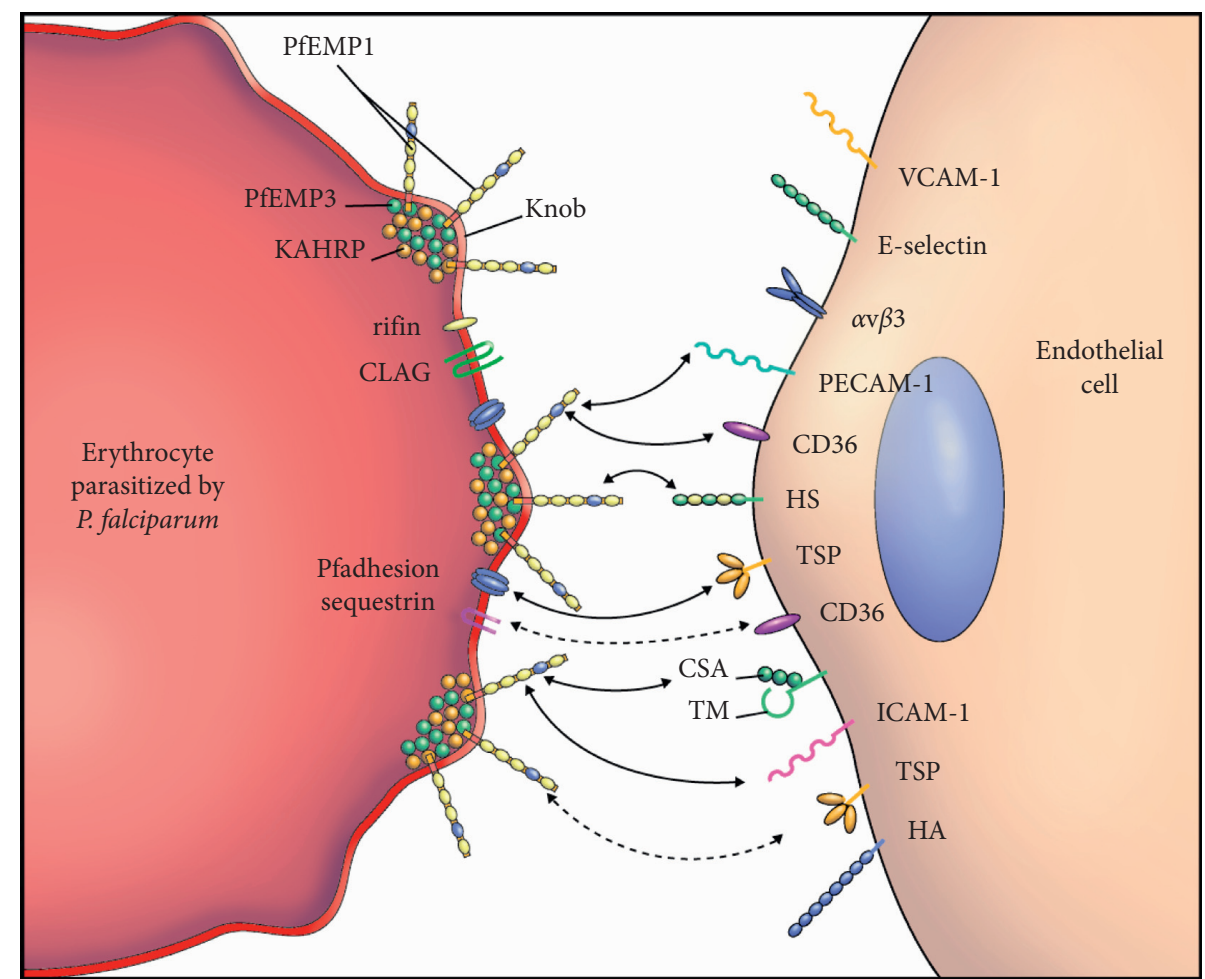

FIGURE 3: Falciparum cytoadherence (VCAM-1: vascular cell adhesion molecule-1, $\alpha v \beta 3$ : integrin, PECAM-1: platelet endothelial cell adhesion molecule-1, HA: hyaluronic acid, CLAG: clag9 ligand) (adopted from [7]).

manifestations of malaria including fever have been largely attributed to the released cytokines and toxins [78, 79].

In addition, the plasmodial DNA presented by hemozoin interacts intracellularly with the Toll-like receptor-9, leading to the release of proinflammatory cytokines that in turn induce cyclooxygenouse (COX-2) upregulating prostaglandins proceeding to induction of fever [80, 81]. Hemozoin has also been linked to induction of apoptosis in 
developing erythroid cells in the bone marrow, thereby causing anemia [82, 83]. Likewise, Mawson hypothesized that the parasites emerge from the liver packed with vitamin $A$ and use retinoic acid as a cell membrane destabilizer to invade the RBCs, causing hemolysis and anemia [84].

The clinical manifestations of severe malaria, caused especially by falciparum, are directly correlated with the induction of strong proinflammatory immune responses [85]. Hyperactive immune response is one of the major contributors to cerebral malaria vasculopathy, and fatal outcome is generally ascribed to sequestration of activated macrophages, parasitized erythrocytes, and platelets in cerebral vessels [86].

\section{Diagnosis of Malaria}

Malaria must be diagnosed early and accurately to end up with an effective management of patients. Broadly, one can classify it into clinical and parasitological diagnoses. Clinical diagnosis is based on the patient's symptoms and on signs at physical examination $[6,13]$.

All of the suspected malaria should be confirmed with a parasitological diagnosis in all settings [13]. Light microscopy and RDTs are routinely employed methods for parasitological diagnosis of malaria. Detection of the parasites on giemsa-stained peripheral blood smears by light microscopy is used as the gold standard for diagnosis of malaria. As knowlesi and malariae have almost similar morphology, microscopy alone is insufficient to diagnose knowlesi $[87,88]$. In case of vivax, ovale, and malariae, all development stages subsequent to the liver cycle can be seen in the peripheral blood. However, in falciparum, only ring forms and banana-like gametocytes are usually present in the peripheral blood since mature parasites become sequestered [89].

In areas where microscopy is not readily available, RDTs can be used and are based on the detection of antigens or enzymatic activities associated with the parasites. The most common antigens for RDTs are $P$. falciparum histidine-rich protein-2 (PfHRP2), specific for falciparum malaria, and two enzymes of the parasite glycolytic pathways, namely plasmodial lactate dehydrogenase (pLDH) and aldolase. LDH can be specific for falciparum or vivax malaria or it can be a variant pan specific (common to all six species). RDTs can also measure parasite antigens when mature parasites are sequestered. But, some isolates from the Amazon region, Africa, and India have been found lacking the PfHRP2, probably HRP2 gene deletion, which threatens the ability to diagnose and appropriately treat people infected with $\mathrm{fal}$ ciparum malaria [88, 89]. In 2005, single-species RDTs were introduced in Ethiopia. Years after, multispecies RDTs are being supplied by FMoH to health posts [90].

PCR-based methods, another parasitological diagnostic means, are the most sensitive test able to identify low levels of parasitemia, parasite species, or mixed infections, but not a suitable method for routine use. A species-specific loopmediated isothermal amplification (LAMP) method has become widely accepted for identifying knowlesi infections. Besides, PCR is helpful as a research tool in epidemiological studies, clinical trials, and for detection of molecular markers of drug resistance to antimalarial agents [11, 91].

The $4^{\text {th }}$ parasitological method is the serology test based on detection of antibodies against malarial parasites, using either indirect immunofluorescence (IFA) or enzyme-linked immunosorbent assay (ELISA). Serology does not detect current infection but rather measures past exposure [6]. Newly developed rolling circle-enhanced enzyme activity detection (REEAD) and micromagnetic resonance reflaxometric (MMR) test are amenable to deployment in field conditions and are highly accurate and cost- and time-effective [92].

Novel malaria diagnostic targets have been searched and include extremely conserved genes of proteins [93]. The most abundant heat-shock protein (HSP), HSP-70, has been investigated as a new diagnostic protein [94]. Another promising targets would be Plasmodium heme detoxification protein (HDP) for all species of Plasmodium, protozoan dihydrofolate reductase (DHFR) to detect falciparum and vivax spp., Glutamate-rich protein (GLURP), and highmobility group box 1 (HMGB1) protein for falciparum diagnosis [95-97]. Recently, one study reported that infection with malaria makes children smell more inviting to Anopheles gambiae mosquitoes, which carry the disease. An increase in discharges of chemicals known as aldehydes accounted for much of the change in attractiveness. The finding might be valuable in the development of latest noninvasive diagnostic methods because it enables to diagnose carriers of malaria parasites using odors even if they do not feel sick to visit a health institution [98].

\section{Preventive and Control Measures of Malaria}

5.1. Vector Control. Many countries are trying to get rid of malaria. In 2017, a total of forty-six countries reported fewer than 10,000 malaria cases [20]. Ethiopia planned to eliminate malaria by 2020 [99]. To do so, vector control is an effective measure that has to be taken. Anopheles mosquitoes can be reduced via the use of insecticide-treated bed nets (ITNs) and indoor spraying of residual insecticides (IRSs). Endophilic mosquitoes are readily controlled by IRSs. In contrast, exophagic/exophilic vectors are best controlled through source reduction (destruction of the breeding sites) and larviciding [16]. Those insecticides in use, mostly within African countries, are pyrethroids (recommended for use only on bed nets), organochlorines (e.g., DDT), organophosphates, and carbamates [100]. The effectiveness of insecticide-based vector control is endangered by malarial mosquitoes developing resistance for the insecticides used. However, long-lasting insecticidal nets (LLINs) remain effective despite resistance. The current WHO advice for resistance management in areas with LLINs is additive spraying, with nonpyrethroids used on a rotational basis [13].

Attractive toxic sugar bait (ATSB) methods are a new form of vector control measure that slays mosquitoes searching for essential sugar sources in the outdoor environment. These approaches uses fruit or flower smell as an attractant, sugar solution as a feeding stimulant, and oral 
toxin to destroy the mosquitoes [101, 102]. Spraying swarms with aerosols is another vector control method that caused an extraordinary reduction in mosquito density [103]. Individual bite protection methods (e.g., insect repellants and protective clothing) have also been used to reduce malaria transmission by mosquitoes [104, 105]. Jaleta et al. (2016) demonstrated that nonhost (chicken) volatiles can provide protection to mosquito-vectored diseases in combination with established control programmes [106].

Majority of malaria vector species display a more diverse behavior, feeding on livestock and humans. Mosquitoes nourishing on livestock could be targeted through treatment of livestock structures (e.g., IRS of cattle sheds) [107]. Direct treatment of cattle with insecticides by dipping, sponging, or spraying has also been shown to kill mosquitoes and to reduce malaria in the human population $[108,109]$. Use of systemic veterinary insecticides that affect the mosquitoes upon blood feeding is another alternative. Ivermectin has been successfully tested in cattle and demonstrated to both killing mosquitoes and shorten the lifespan of the survivors $[110,111]$.

Remarkable ways to interfere transmission include disrupting steroid hormone signaling in mosquitoes [112], use of transgenic mosquitoes [113, 114], using of paratransgenesis for delivering anti-Plasmodium effector molecules [115], and/or transinfection of mosquitoes with symbiotic bacteria and fungi [116]. The use of a transgenic procedure can improve the sterile insect technique (SIT) for Anopheles induced by radiation [117].

Uniquely, some genetic abnormalities (host polymorphism) in RBCs confer resistance to malaria. This include, among others, deficiency in pyruvate kinase, polymorphic glycophorins, ovalocytosis, spherocytosis, elliptocytosis, sickle-cell traits, thalassaemia traits, and glucose-6-phosphate dehydrogenase (G6PD) deficiency [118-120]. The study of these protective polymorphisms can provide clues concerning naturally occurring systems of host defense, which could be used to develop new therapeutic drugs to combat malaria [119].

Drugs also play a major role in preventing malaria transmission. Intermittent Preventive Therapy (IPT) using sulfadoxine-pyrimethamine (SP) is another malaria control tool in pregnant women, infants, and preschool children where transmission is seasonal $[66,121]$. Repeated Ivermectin Mass Drug Administration (MDA) could also help to control transmission [122]. Chemoprophylactic drugs such as chloroquine, mefloquine, and doxycycline or the combination of atovaquone and proguanil (Malarone ${ }^{\circledR}$ ) are usually used to prevent infection in travelers as they move from no-malaria areas to places where malaria is common [123].

Cotrimoxazole is being administered to patients with human immuno virus (HIV) to guard against Pneumocystis jirovecii pneumonia and has been shown to reduce malaria infections and is a potential candidate for use as prevention in HIV-uninfected pregnant women or children [124]. Methylene blue is an old parasiticidal agent with blood stage activity and has added benefit due to effects against mature male and female falciparum gametocytes [125]. Despite the dearth of new compounds in development for chemoprevention only, DSM-265 demonstrates its potential as a prophylactic drug for travelers [126]. Note that blood-stage parasites surrounded by PVM proliferate within RBCs, and following each cycle of intracellular development, first rupture the PVM using SUB1 and then the RBC membrane through serine repeat antigen protease-like protein (SERA6) to allow egress of the merozoites, which invade fresh RBCs. Compounds that inhibit these proteases would target consecutive, interdependent steps in the egress pathway and so could form a new class of drug intended to prevent parasite proliferation and disease $[39,127]$.

5.2. Malaria Vaccine. The emergence and spread of drug and insecticide resistance has been limiting the current malaria control measures, thus safe and effective vaccine is required to achieve the world malaria eradication programme objectives. The justification for a malaria vaccine development is the observation that people living in endemic areas develop clinical protective immunity despite the morphological changes and antigenic variations during the parasite life cycle allows them to escape the protective immune responses of the host [128]. So far, three types of vaccine candidates have been intensively investigated: pre-erythrocytic vaccines to prevent blood-stage infection; blood-stage vaccines to clear parasitaemia and prevent clinical disease; and transmission-blocking vaccines to prevent infection of mosquitoes and interrupt malaria transmission in populations [129].

Pre-erythrocytic vaccines target the sporozoites and/or hepatic stages of the parasite. Some vaccines of this group are RTS,S/AS01 in phase IV clinical trial, falciparum sporozoite vaccine (PfSPZ) in phase II trials [130], vivax malaria protein 1 (VMP001/AS01B) in phase I/IIa trial [131], cell-traversal protein for ookinetes and sporozoites (CelTOS) [FMP012/ GLA-SE or AS01] under phase I/IIa clinical trial [132], and vaccine of chimpanzee adenovirus expressing CS (CSVAC) [133], genetically attenuated parasite (GAP) vaccines and chemoprophylaxis vaccination $(\mathrm{CVac})$ in Phase I clinical trial [130]. Using in vitro studies, falciparum liver-stage antigens (PfLSA-1, 2 \& 3) and vivax liver-stage antigens (PvLSAs) are recognized as a novel candidate vaccine targeting infected hepatocytes [134, 135].

Several asexual blood stage vaccines, most target merozoite antigens, are in clinical researches. Candidates for erythrocyte-stage vaccine are AMA1 [136], erythrocytebinding antigen (EBA-175) [137], MSP-1 [138], MSP-1 19 [139], MSP-2 [140], MSP-3 [96], and serine repeat antigen 5 (SERA5) [141]. None has resulted in clear clinical protection, probably due to the highly polymorphic nature of the vaccine structures [142]. But, efforts to enhance the efficacy either with a novel adjuvants [143] using viral vector primeboost strategies [144] or by combining AMA1 and MSP1 [145] have been increasing even though new nonpolymorphic falciparum ligands, CX3CL1-binding proteins (CBP1 and CBP2), are now revealed by Hermand and his coworkers, which provides a new opportunity for innovative vaccination approaches [146]. With further research, new 
antigens, falciparum reticulocyte-binding protein homolog 5 (PfRH5) and rhoptry-associated leucine zipper-like protein 1 (RALP1) having a potential to become blood-stage vaccine candidates, are being discovered $[147,148]$. PfRH5 is actually in phase I clinical study [130]. Currently, falciparum merozoite protein MSP4 (naturally induces a strong antibody response in malaria endemic areas) has created an interest to be included into candidate vaccines [149]. Multigravidae who have acquired antibodies against VAR2CSA are indeed protected from pregnancy-associated malaria after one pregnancy. Based on this finding, vaccine candidates directed against VAR2CSA are under optimization [150].

Transmission-blocking vaccines (TBVs) target surface proteins expressed on gametocytes, zygotes, and ookinetes to prevent parasite development in the mosquito mid gut by specific host antibodies, complement proteins, and cytokines [151]. The vaccine candidates in this group include the gametocyte antigens (Pfs48/45 and Pfs230) [152], falciparum ookinete surface antigens (Pfs25 and Pfs28) [153], and their vivax homologues Pvs25 and Pvs28 [154]. Other more recently known targets of curiosity include Pfs47 (implicated in parasite immune evasion in the mosquito vector) [155] and PfHAP2 which are expressed on the male gametocyte and microgamete [156]. Different vaccines with their formulation concerns, classified within the above-mentioned three vaccine categories and beyond the scope of this article, are discussed in detail elsewhere $[132,157]$.

\section{Treatment of Malaria}

6.1. Traditional Medicine. Traditional medicine (TM) use varies among countries depending on a number of factors. In Singapore and the Republic of Korea where the conventional health-care system is quite well established, 76\% and $86 \%$ of the respective populations still commonly use TMs. About $90 \%$ of general hospitals provide TM services for both outpatients and inpatients in China. Over 100 million Europeans are TM users [158]. In developing countries, $80 \%$ of the people almost exclusively use TMs. Virtually, $80 \%$ of the population living in Ethiopia is dependent on traditional medicine which essentially involves the use of plants $[159,160]$.

More than 1,200 plants that possess antimalarial activities are reported worldwide [161]. For example, Ampelozyziphus amazonicus and Strychnopsis thouarsii were commonly used in malaria-endemic areas of Brazil and Madagascar, and their antisporozoite activities have been demonstrated. It is probable that some of antimalarial plants contain as yet undiscovered active constituents [162].

Ethiopia is rich in a wide range of tropical habitats, remarkable biodiversity, and use of traditional remedies for the treatment of various ailments [163]. Studies conducted on numerous traditionally claimed Ethiopian medicinal plants confirmed their antimalarial activities including Phytolacca dodecandra [164], Justicia schimperiana [165], Artemisia abyssinica [166], Vernonia amygdalina [167], Buddleja Polystachya [168], Strychnos mitis [169], Echinops kebericho [170], Aloe trichosantha, Cadaba rotundifolia
[171], Adhatoda schimperiana, Piper capense [172], and Gardenia ternifolia [173].

6.2. Conventional Medicine. Malaria can lead to fatal outcomes in only few days, thus treatment should be started as soon as possible. The main targets of current antimalarial drugs are asexual blood stages of the parasite, responsible for the malarial symptoms [89]. Nowadays, the available antimalarials can be grouped into five classes according to their chemical structure and biological activity: (i) quinolinebased antimalarials: 4-aminoquinolines (chloroquine, amodiaquine, and piperaquine) and 8-aminoquinolines (premaquine and tafenoquine); (ii) arylaminoalcohols-quinine, mefloquine, halofantrine, and lumefantrine; (iii) antifolate compounds (pyrimethamine, proguanil, dapsone, and sulfadoxine); (iv) artemisinin and its derivatives: first generation (dihdyroartemisinin, artesunate, arteether, and artemether) and second generation (artemisone); and (v) hydroxynapthoquinone-atovaquone $[89,174]$.

Chloroquine is a blood schizonticidal agent and the drug of choice for all malarial parasites except for chloroquineresistant Plasmodium strains. Although almost all strains of malariae are susceptible, falciparum, vivax, and even some ovale strains have been reported as resistant to chloroquine [35]. Chloroquine resistance for falciparum is due to point mutations in the gene encoding chloroquine resistance transporter (PfCRT) protein, resulting in reduced drug accumulation in the food vacuole [175]. Drug resistance to chloroquine has been reported in Ethiopia [89]. Amodiaquine is effective against some parasite strains that are resistant to chloroquine, although some cross resistance exists [176]. Piperaquine also has an excellent activity on chloroquine-resistant species [177].

Primaquine (tissue schizonticidal agent) is effective against the hypnozoites of vivax and ovale malaria and can kill gametocytes and consequently block the malaria transmission. Therefore, its effect on oocyst and sporozoite formation (and thus onward transmission of treated infection) precedes its effect on gametocytes carriage. It has weak activity against the asexual blood stage of vivax malaria. Primaquine is indeed used to achieve complete elimination of relapsing malaria due to vivax or ovale, in combination with a blood schizontocide for the erythrocytic parasites [12].

Quinine kills large ring and tropozoite asexual parasites and is gametocidal against vivax, ovale, and malariae but not falciparum malaria [178]. Mefloquine is also a blood schizonticide, active against the erythrocytic stages of all malaria parasites [179]. Proguanil is a biguanide compound that is active against all stages of Plasmodium [180]. SP has been the drug of choice for IPT in first and second trimester pregnancy and in infants living within malaria-endemic areas [181]. In combination with amodiaquine, SP is also used for seasonal malaria chemoprevention in children [12]. It is active predominantly against later development stages of asexual parasites. Resistance is caused by point mutations in parasite enzymes namely, dihydropteroate synthase (DHPS) 
and DHFR [182]. Atovaquone is active against all Plasmodium species. It is ubiquinone analogue and acquires resistance related to a single mutation of cytochrome $b$ gene of the parasite [183].

Artemisinin (endoperoxide sesquiterpene lactone) is a potent and fast acting blood schizonticidal killing all parasite stages. Falciparum resistance to them has now been detected in 5 countries in the Greater Mekong subregion: Cambodia, Lao People's Democratic Republic, Myanmar, Thailand, and Vietnam. These resistant strains have the capacity to spread to different parts of the world including Ethiopia and to subsequently become a global threat for malaria control and treatment $[12,184]$.

WHO recommends artemisinin-based combination therapies (ACT) for the treatment of uncomplicated malaria caused by falciparum parasite or by chloroquine-resistant vivax, ovale, malariae, and knowlsi. Atovaquone-proguanil may be considered for the treatment of uncomplicated malaria in travelers outside malaria-endemic areas. Quinine plus clindamycin is used for uncomplicated malaria treatment in the first trimester of pregnancy [12]. In Ethiopia, artemether-lumefantrine $\left(\right.$ Coartem $\left.^{\mathrm{TM}}\right)$ is suggested as the first-line drug for uncomplicated falciparum malaria and chloroquine for other species, but oral quinine is considered as a second option [90].

More recently, injectable artesunate has become the treatment of choice for severe malaria worldwide in infants, children, lactating women, and pregnant women in all trimesters. After $24 \mathrm{~h}$, the treatment should be completed with oral ACT [12]. Quinidine plus doxycycline, tetracycline, or clindamycin is the preferred drug in the USA to treat severe malaria [185]. Coming to Ethiopia, injectable artesunate is the preferred drug, and intramuscular artemether is an alternative drug. If these two drugs are not available, injectable quinine can be used to manage severe malaria [90].

6.3. Drugs in the Pipeline. The development of resistant strains and lack of new drugs are the limiting aspects in the fight against malaria. These factors trigger the continuing need for research of new classes of antimalarial agents and a re-examination of the existing antimalarial drugs. Hence, ozonides (synthetic peroxides) are proved to be useful substitutes for artemisinin. The first-generation ozonide OZ277 subsequently called arterolane was developed through a partnership between Medicines for Malaria Venture (MMV) and Ranbaxy. In 2012, Ranbaxy launched the combination of arterolane maleate and piperaquine phosphate as a 3-day treatment in India. After a limited Phase III programme, the combined drug has got approval under the trade name Synriam in India in 2013, followed by approval in seven African Nations in 2014 [129, 186].

Multiple novel combination therapies, including azithromycin-chloroquine [187], pediatric pyronaridine-artesunate, pediatric dihydroartemisin in piperaquine [188], and trimethoprim-sulfamethoxazole [129] are in phase III trial. Tafenoquine (primaquine analog) is currently being tested in pivotal Phase III trial and has proven activity against hypnozoites [189]. It has the same G6PD deficiency liability, but has the advantage of being a single-dose treatment [190] and possesses higher activity than primaquine [191].

Many new drugs are in phase 2 clinical development. The second-generation peroxide OZ439 (artefenomel) which has blood stage activity for falciparum and vivax malaria [190] is being tested in Phase IIb combination trial with piperaquine and is also tested with ferroquine (4-aminoquinoline), both in partnership between MMV and Sanofi [129]. The new organometallic drug ferroquine (SR97193) was found to be active against chloroquine-resistant strains and is currently undergoing Phase II clinical trials as combination therapy with artesunate [190]. Novartis currently has two new antimalarials, KAE609 (Cipargamin) and KAF156, in Phase II clinical testing. KAE609 (belongs to spiroindolone) has an inhibitory effect on falciparum cation channel (PfATPase4). It is blood schizonticide for falciparum and vivax. KAF156 (class of imidazolopiperazines) is with potential to treat and prevent malaria since it acts at multistage of the parasite life cycle $[192,193]$. This drug is now joined phase IIb trial in combination with lumefantrine $[194,195]$.

DSM265 is another compound in phase II trial that inhibits falciparum dihydroorotate dehydrogenase $(\mathrm{PfDHODH})$ and vivax dihydroorotate dehydrogenase (PvDHODH) enzymes [195]. This new chemical is a long acting with blood and liver stage activity [193]. Artemisone, a drug in Phase II study, provides a single-dose cure in Aotus monkeys infected with falciparum malaria at $10 \mathrm{mg} / \mathrm{kg}$ when combined with mefloquine $5 \mathrm{mg} / \mathrm{kg}$ [196]. Fosmidomycin, a natural antibacterial agent that inhibits an enzyme involved in the synthesis of isoprenoids (1-deoxy-D-xylulose 5phosphate reductoisomerase) [161], is under combination therapy trial with piperaquine in phase II [188] in order to kill blood schizonts of uncomplicated falciparum malaria [191]. AQ-13, a modified chloroquine in phase II [186], retains activity against chloroquine-resistant parasites [197].

A phenothiazine derivative methylene blue is being developed (phase II) in combination with artesunateamodiaquine as a strategy to protect against emergence of artemisinin resistance secondary to its falciparum schizonticidal effect and reduce transmission owing to gametocytocidal activity. Methylene blue acts by inhibiting falciparum glutathione reductase and as a result, prevents haem polymerization [126]. Polysaccharide heparin analogue Sevuparin (DF02) which is taken as an adjunctive therapy retains the antiadhesive effects of heparin without the antithrombin properties and has been shown to block merozoite invasion, cytoadherence, and rosetting [198]. MMV39048 is an aminopyridine currently in Phase 2a (NCT02880241) trial, and its target was identified to be lipid phosphatidylinositol 4-kinase (PfPI4K). This drug has destructive activity on multiple stages of the parasite with possible efforts for chemoprevention [193, 199]. Albitiazolium (T3/SAR97276) has also reached Phase II clinical study. It acts mainly by deterring the transport of choline into the parasite [200].

An additional treatment panorama that has recently been entered into human clinical trial is quinoline-4-carboxamide DDD107498. It is an inhibitor of peptide elongation factor 2 with activity against pre-erythrocytic and 
blood stages as well as mature gametocytes [201, 202]. A dihydroisoquinolone, SJ733, which inhibits gametocytogenesis as well as blood schizonts for falciparum and vivax, is now in human trial. It binds to a malaria parasite protein that serves as a sodium pump to interfere with the protein and sodium ions build up [203, 204]. Additionally, CDRI 97/ 78 (trioxane), ACT-451840 (phenylalanine-based compound), P218 (diaminopyridine and DHFR inhibitor), and GSK369796 (N-tert-butyl isoquine) are also among drugs under phase I study $[129,193]$.

Very recent analysis has predicted that lead compounds in a preclinical study have $8 \%$ possibility to become a registered product [205]. Most of them (e.g., SAR121, DM1157, and AN 13762) are blood schizonticidal except UCT 943 and NPC1161B, which has multistage activity [193]. Triaminopyrimidine MMV 253 that inhibits Plasmodium ATPase and aminomethylphenol JPC-3210, active against multidrug resistant falciparum, are long-acting agents in an early preclinical experiment [206, 207]. SC83288 (an amicarbalide derivative) is the only agent in preclinical investigation that are going to treat sever malaria [208]. It is also possible to list Genz-668764, ML238, ACT213615, and TDR84420 within the new chemical entity group [209].

Furthermore, a pyrazoleamide 21A092 which targets sodium channel (ATPase4) like KAE609 and SJ557733 is in preclinical discovery phase [210]. Dantrolene was identified as a novel inhibitor of plasmodial surface anion channel (PSAC), and it may be a lead compound for antimalarial drug development [211]. Acridinones such as WR249685 and T3.5, a new class of selective malaria parasite mitochondrial $b c 1$ inhibitors, had a great potential to become novel antimalarial drugs $[212,213]$. The study conducted by Wilson et al. (2015) identified for the first time that macrolide antibiotics inhibit Plasmodium species merozoite invasion into erythrocytes in vitro. This result directs the development of safe and effective macrolide antibiotics with dual modalities to combat malaria and reduce the parasite's options for resistance [214]. Antiadhesion adjunctive therapies including levamisole are under research in the laboratory [69]. In vivo and in vitro studies showed that an acriflavine (antibacterial and anticancer drug) impairs DNA replication foci formation in Plasmodium berghei and affects the enzymatic activities of apicoplast-specific Gyrase protein. This interesting work tells us the potential of this old drug to become a future antimalarial drug [215]. Now, a team of researchers has discovered thioredoxin enzymes which are different from the human one but critical for the survival of plasmodium by balancing redox state inside the parasite. So, the team is working with industry partners to create new drugs which will effectively target this enzyme and kill the parasite without affecting the human host [216].

\section{Conclusion}

Malaria, an ancient human disease, remains an important cause of illness and death in children as well as adults in endemic countries. Falciparum and vivax malaria produce a big challenge in the health of the community. Regardless of decrement in prevalence and incidence of malaria, its transmission is still dynamic around the world. Therefore, malaria control requires an integrated approach including prevention primarily vector control and prompt treatment with effective antimalarial agents. However, an increasing resistance towards control measures and the currently available antimalarial drugs is a challenge to fight against malaria. Despite decades of intense research, no licensed malaria vaccines are available until now. Although many drugs are in the pipeline, most of them are not able to kill both gametocytes and hypnozoites. If the past instance is an indicator, resistance to the conventional antimalarials will spread to Africa including Ethiopia. Success and resistance are creating malaria scenery that requires new tools and approaches. Thus, the globe is in an urgent need of new, safe, and effective insecticides and drugs, as well as vaccines that can take over the currently resistance-prone phenomenon. In Ethiopia, a lot has to be performed to forward the malaria elimination plan.

\section{Conflicts of Interest}

The authors declare that there are no conflicts of interest regarding the publication of this paper.

\section{References}

[1] W. Service and H. Townson, "The anopheles vector," in Essential Malariology, H. Gilles and D. Warrell, Eds., pp. 59-84, Arnold, London, UK, 4th edition, 2002.

[2] R. Carter and K. N. Mendis, "Evolutionary and historical aspects of the burden of malaria," Clinical Microbiology Reviews, vol. 15, no. 4, pp. 564-594, 2002.

[3] B. M. Greenwood, K. Bojang, C. J. Whitty, and G. A. Targett, "Malaria," The Lancet, vol. 365, no. 9469, pp. 1487-1498, 2005.

[4] NIAID, "Understanding malaria," Fighting an Ancient Scourge, No. 07-7139, U.S. Dept. of Health and Human Services, National Institutes of Health, National Institute of Allergy and Infectious Diseases, Bethesda, MD, USA, 2007.

[5] A. U. Krettli and L. H. Miller, "Malaria: a sporozoite runs through it," Current Biology, vol. 11, no. 10, pp. R409-R412, 2001.

[6] CDC, The History of Malaria, an Ancient Disease, Division of Parasitic Diseases and Malaria, USA, 2016.

[7] S. David, History, Lifecycle, Epidemiology, Pathology, and Control; Malariology Overview, Johns Hopkins University, Baltimore, MD, USA, 2006.

[8] L. J. Bruce-Chwatt, “Alphonse Laveran's discovery 100 years ago and today's global fight against malaria," Journal of the Royal Society of Medicine, vol. 74, no. 7, pp. 531-536, 1981.

[9] J. M. Chavatte, F. Chiron, A. Chabaud, and I. Landau, "Fidélisation du couple hôte-vecteur facteur probable de spéciation: 14 espèces dePlasmodiumde la Pie," Parasite, vol. 14, no. 1, pp. 21-37, 2007.

[10] F. E. Cox, "History of the discovery of the malaria parasites and their vectors," Parasites \& Vectors, vol. 3, no. 1, p. 5, 2010.

[11] G. Snounou, S. Viriyakosol, W. Jarra, S. Thaithong, and K. N. Brown, "Identification of the four human malaria parasite species in field samples by the polymerase chain reaction and detection of a high prevalence of mixed 
infections," Molecular and Biochemical Parasitology, vol. 58, no. 2, pp. 283-292, 1993.

[12] WHO, Guidelines for the Treatment of Malaria, WHO, Geneva, Switzerland, 3rd edition, 2015.

[13] WHO, World Malaria Report, WHO, Geneva, Switzerland, 2015.

[14] FMoH, An Epidemiological Profile of Malaria in Ethiopia, Ministry of Health, Federal Democratic Republic of Ethiopia, Addis Ababa, Ethiopia, Version 1.0, 2014.

[15] R. Harbach, "The phylogeny and classification of Anopheles," in Anopheles Mosquitoes-New Insights into Malaria Vectors, S. Manguin, Ed., Intecth, London, UK, 2013.

[16] CDC, Anopheles Mosquitoes, Division of Parasitic Diseases and Malaria, USA, 2015.

[17] A. Owusu-Ofori, C. Parry, and I. Bates, "Transfusiontransmitted malaria in countries where malaria is endemic: a review of the literature from sub-Saharan Africa," Clinical Infectious Disease, vol. 51, no. 10, pp. 1192-1198, 2010.

[18] President's Malaria Initiative Ethiopia (PMI), Malaria Operational Plan FY 2014, 2014.

[19] RBMP, Evidence for Advocacy: Key Statistics on the Fight against Malaria, RBMP, Geneva, Switzerland, 2015.

[20] WHO, World Malaria Report, WHO, Geneva, Switzerland, 2018.

[21] M. Ananya, Malaria Epidemiology. Reviewed by April Cashin-Garbutt, BA Hons (Cantab) Pdf, http://www.newsmedical.net/health/, 2013.

[22] CDC Global Health-Ethiopia, Centers for Disease Control and Prevention, Center for Global Health, Atlanta, USA, 2016.

[23] Index Mundi, Ethiopia Major Infectious Diseases, https:// www.indexmundi.com/ethiopia/major_infectious_diseases. html, 2018

[24] S. Ayalew, H. Mamo, A. Animut, and B. Erko, "Assessment of current malaria status in light of the ongoing control interventions, socio-demographic and environmental variables in Jiga area, northwest Ethiopia," PLoS One, vol. 11, no. 1, Article ID e0146214, 2016.

[25] A. Yibeltal, M. Abeba, B. Abebaw, K. Bekalu, and T. Asmare, "Prevalence of malaria and associated risk factors among asymptomatic migrant laborers in West Armachiho District, Northwest Ethiopia," Research and Reports in Tropical Medicine, vol. 9, pp. 95-101, 2018.

[26] D. Ayele, T. Zewotir, and H. Mwambi, "The risk factor indicators of malaria in Ethiopia," International Journal of Medicine and Medical Sciences, vol. 5, no. 7, pp. 335-347, 2013.

[27] A. Deribew, T. Dejene, B. Kebede et al., "Incidence, prevalence and mortality rates of malaria in Ethiopia from 1990 to 2015: analysis of the global burden of diseases 2015," Malaria Journal, vol. 16, p. 271, 2017.

[28] WHO, Training Module on Malaria Control Malaria Entomology and Vector Control. Guide for Participants, WHO, Geneva, Switzerland, 2013.

[29] T. Ricardo, K. Parisa, A. Katherine, and G. Douglas, "Plasmodium life cycle and the pathogenesis of malaria. From innate sensing of malaria parasites," Nature Reviews Immunology, vol. 14, pp. 744-757, 2014.

[30] N. F. Walker, B. Nadjm, and C. J. M. Whitty, "Malaria," Medicine, vol. 38, no. 1, pp. 41-46, 2010.

[31] P. Szmitko, M. L. Kohn, and A. E. Simor, "Plasmodium falciparum malaria occurring eight years after leaving an endemic area," Diagnostic Microbiology and Infectious Disease, vol. 61, no. 1, pp. 105-107, 2008.
[32] C. Theunissen, P. Janssens, A. Demulder et al., "Falciparum malaria in patient 9 years after leaving malaria-endemic area," Emerging Infectious Diseases, vol. 15, no. 1, pp. 115-116, 2009.

[33] I. Poilane, V. Jeantils, and L. Carbillon, "Découverte fortuite de paludisme à Plasmodium falciparum au cours de la grossesse: à propos de deux cas," Gynécologie Obstétrique \& Fertilité, vol. 37, no. 10, pp. 824-826, 2009.

[34] W. Jiraprapa, E. Salenna, X. Huji, and Michael Food, "Immunity to asexual blood stage malaria and vaccine approaches," Immunology and Cell Biology, vol. 80, pp. 401-414, 2002.

[35] D. Bhowmik, B. Chiranjib, N. Singh, J. Jaiswal, and K. P. Kumar, "Recent advances in prevention, treatment and medication of malaria," Journal of Chemical and Pharmaceutical Research, vol. 2, no. 1, pp. 83-90, 2010.

[36] W. B. Gratzer and A. R. Dluzewski, "The red blood cell and malaria parasite invasion," Seminars in Hematology, vol. 30, no. 30, pp. 232-247, 1993.

[37] C. John, Biology of Malaria Parasites, Malaria Parasites, O. Okwa, Ed., InTech, London, UK, 2012, ISBN: 978-953-510326-4.

[38] L. H. Bannister, J. M. Hopkins, R. E. Fowler, S. Krishna, and G. H. Mitchell, "A brief illustrated guide to the ultrastructure of Plasmodium falciparum asexual blood stages," Parasitology Today, vol. 16, no. 10, pp. 427-433, 2000

[39] A. F. Cowman, J. Healer, D. Marapana, and K. Marsh, "Malaria: biology and disease," Cell, vol. 167, no. 3, pp. 610-624, 2016.

[40] K. J. Robson, U. Frevert, I. Reckmann et al., "Thrombospondin-related adhesive protein (TRAP) of Plasmodium falciparum: expression during sporozoite ontogeny and binding to human hepatocytes," The EMBO Journal, vol. 14, no. 16, pp. 3883-3894, 1995.

[41] U. Frevert, P. Sinnis, C. Cerami, W. Shreffler, B. Takacs, and V. Nussenzweig, "Malaria circumsporozoite protein binds to heparan sulfate proteoglycans associated with the surface membrane of hepatocytes," The Journal of Experimental Medicine, vol. 177, no. 5, pp. 1287-1298, 1993.

[42] S.-J. Cha, K. Park, P. Srinivasan et al., "CD68 acts as a major gateway for malaria sporozoite liver infection," The Journal of Experimental Medicine, vol. 212, no. 9, pp. 1391-1403, 2015.

[43] O. Silvie, E. Rubinstein, J.-F. Franetich et al., "Hepatocyte CD81 is required for Plasmodium falciparum and Plasmodium yoelii sporozoite infectivity," Nature Medicine, vol. 9, no. 1, pp. 93-96, 2003.

[44] M. R. Baldwin, X. Li, T. Hanada, S.-C. Liu, and A. H. Chishti, "Merozoite surface protein 1 recognition of host glycophorin A mediates malaria parasite invasion of red blood cells," Blood, vol. 125, no. 17, pp. 2704-2711, 2015.

[45] A. F. Cowman, D. Berry, and J. Baum, "The cellular and molecular basis for malaria parasite invasion of the human red blood cell," The Journal of Cell Biology, vol. 198, no. 6, pp. 961-971, 2012.

[46] M. A. Boston, Malaria Parasite's Essential Doorway into Red Blood Cells Illuminated, http://www.hsph.harvard.edu/news, 2016.

[47] G. H. Mitchell, A. W. Thomas, G. Margos, A. R. Dluzewski, and L. H. Bannister, "Apical membrane antigen 1, a major malaria vaccine candidate, mediates the close attachment of invasive merozoites to host red blood cells," Infection and Immunity, vol. 72, no. 1, pp. 154-158, 2004. 
[48] P. Sharma and C. E. Chitnis, "Key molecular events during host cell invasion by Apicomplexan pathogens," Current Opinion In Microbiology, vol. 16, no. 4, pp. 432-437, 2013.

[49] D. C. G. Mayer, J. Cofie, L. Jiang et al., "Glycophorin B is the erythrocyte receptor of Plasmodium falciparum erythrocytebinding ligand, EBL-1," Proceedings of the National Academy of Sciences, vol. 106, no. 13, pp. 5348-5352, 2009.

[50] M. L. Tonkin, M. Roques, M. H. Lamarque et al., "Host cell invasion by apicomplexan parasites: insights from the costructure of AMA1 with a RON2 peptide," Science, vol. 333, no. 6041, pp. 463-467, 2011.

[51] E. S. Zuccala and J. Baum, "Cytoskeletal and membrane remodelling during malaria parasite invasion of the human erythrocyte," British Journal of Haematology, vol. 154, no. 6, pp. 680-689, 2011.

[52] D. T. Riglar, D. Richard, D. W. Wilson et al., "Super-resolution dissection of coordinated events during malaria parasite invasion of the human erythrocyte," Cell Host \& Microbe, vol. 9, no. 1, pp. 9-20, 2011.

[53] T. Y. Sam-Yellowe, "Rhoptry organelles of the apicomplexa: their role in host cell invasion and intracellular survival," Parasitology Today, vol. 12, no. 8, pp. 308-316, 1996.

[54] M. Aikawa, L. Miller, J. Johnson, and J. Rabbege, "Erythrocyte entry by malarial parasites. A moving junction between erythrocyte and parasite," The Journal of Cell Biology, vol. 77, no. 1, pp. 72-82, 1978.

[55] S. Besteiro, J.-F. Dubremetz, and M. Lebrun, "The moving junction of apicomplexan parasites: a key structure for invasion," Cellular Microbiology, vol. 13, no. 6, pp. 797-805, 2011.

[56] H. E. Bullen, C. J. Tonkin, R. A. O’Donnell et al., “A novel family of Apicomplexan glideosome-associated proteins with an inner membrane-anchoring role," Journal of Biological Chemistry, vol. 284, no. 37, pp. 25353-25363, 2009.

[57] E. Ryan, "Malaria: epidemiology, pathogenesis, diagnosis, prevention, and treatment-an update," Current Clinical Topics In Infectious Disease, vol. 21, pp. 83-113, 2001.

[58] A. Craig and A. Scherf, "Molecules on the surface of the Plasmodium falciparum infected erythrocyte and their role in malaria pathogenesis and immune evasion," Molecular and Biochemical Parasitology, vol. 115, no. 2, pp. 129-143, 2001.

[59] S. Kyes, P. Horrocks, and C. Newbold, "Antigenic variation at the infected red cell surface in malaria," Annual Review of Microbiology, vol. 55, no. 1, pp. 673-707, 2001.

[60] A. Scherf, J. J. Lopez-Rubio, and L. Riviere, "Antigenic variation in Plasmodium falciparum," Annual Review of Microbiology, vol. 62, no. 1, pp. 445-470, 2008.

[61] B. O. Carvalho, S. C. P. Lopes, P. A. Nogueira et al., "On the cytoadhesion of Plasmodium vivax-infected erythrocytes," The Journal of Infectious Diseases, vol. 202, no. 4, pp. 638$647,2010$.

[62] A. G. Maier, M. Rug, M. T. O’Neill et al., "Exported proteins required for virulence and rigidity of Plasmodium falciparum-infected human erythrocytes," Cell, vol. 134, no. 1, pp. 48-61, 2008.

[63] H. May and J. Nicholas, "Molecular mechanisms of cytoadherence in malaria," American Journal of Physiology, vol. 276, no. 6, pp. C1231-C1242, 1999.

[64] S. J. Chakravorty and A. Craig, "The role of ICAM-1 in Plasmodium falciparum cytoadherence," European Journal of Cell Biology, vol. 84, no. 1, pp. 15-27, 2005.

[65] A. F. Sander, A. Salanti, T. Lavstsen et al., "Positive selection of Plasmodium falciparum parasites with multiple var2csa- type PfEMP1 genes during the course of infection in pregnant women," The Journal of Infectious Diseases, vol. 203, no. 11, pp. 1679-1685, 2011.

[66] A. Bardají, Q. Bassat, P. L. Alonso, and C. Menéndez, "Intermittent preventive treatment of malaria in pregnant women and infants: making best use of the available evidence," Expert Opinion on Pharmacotherapy, vol. 13, no. 12, pp. 1719-1736, 2012.

[67] N. Rasti, M. Wahlgren, and Q. Chen, "Molecular aspects of malaria pathogenesis," FEMS Immunology \& Medical Microbiology, vol. 41, no. 1, pp. 9-26, 2004.

[68] Q. Chen, A. Barragan, V. Fernandez et al., "Identification of Plasmodium falciparum erythrocyte membrane protein 1 (PfEMP1) as the rosetting ligand of the malaria parasite $P$. falciparum," The Journal of Experimental Medicine, vol. 187, no. 1, pp. 15-23, 1998.

[69] J. A. Rowe, A. Claessens, R. A. Corrigan, and M. Arman, "Adhesion of Plasmodium falciparum-infected erythrocytes to human cells: molecular mechanisms and therapeutic implications," Expert Reviews In Molecular Medicine, vol. 11, p. e16, 2009.

[70] A. M. Vogt, A. Barragan, Q. Chen, F. Kironde, D. Spillmann, and M. Wahlgren, "Heparan sulfate on endothelial cells mediates the binding of Plasmodium falciparum-infected erythrocytes via the DBL1a domain of PfEMP1," Blood, vol. 101, no. 6, pp. 2405-2411, 2003.

[71] A. Barragan, P. G. Kremsner, M. Wahlgren, and J. Carlson, "Blood group A antigen is a coreceptor in Plasmodium falciparum rosetting," Infection and Immunity, vol. 68, no. 5, pp. 2971-2975, 2000.

[72] R. Udomsanpetch, K. Thanikkul, S. Pukrittayakamee, and N. J. White, "Rosette formation by Plasmodium vivax," Transactions of the Royal Society of Tropical Medicine and Hygiene, vol. 89, no. 6, pp. 635-637, 1995.

[73] B. J. Angus, K. Silamut, R. Udomsangpetch, K. Thanikkul, and N. J. White, "Short report: rosette formation in Plasmodium ovale infection," The American Journal of Tropical Medicine and Hygiene, vol. 55, no. 5, pp. 560-561, 1996.

[74] O. K. Doumbo, C. V. Plowe, K. E. Lyke et al., "High levels of Plasmodium falciparum rosetting in all clinical forms of severe malaria in African children," The American Journal of Tropical Medicine and Hygiene, vol. 81, no. 6, pp. 987-993, 2009.

[75] P. McQueen and F. McKenzie, "Age-structured red blood cell susceptibility and the dynamics of malaria infections," Proceedings of the National Academy of Sciences United States of America, vol. 101, no. 24, pp. 9161-9166, 2003.

[76] G. Krishnegowda, A. M. Hajjar, J. Zhu et al., "Induction of proinflammatory responses in macrophages by the glycosylphosphatidylinositols of Plasmodium falciparum," Journal of Biological Chemistry, vol. 280, no. 9, pp. 8606-8616, 2005.

[77] S. Das, N. Hertrich, A. J. Perrin et al., "Processing of Plasmodium falciparum merozoite surface protein MSP1 activates a spectrin-binding function enabling parasite egress from RBCs," Cell Host \& Microbe, vol. 18, no. 4, pp. 433-444, 2015.

[78] C. Coban, K. J. Ishii, T. Kawai et al., "Toll-like receptor 9 mediates innate immune activation by the malaria pigment hemozoin," The Journal of Experimental Medicine, vol. 201, no. 1, pp. 19-25, 2005.

[79] I. A. Clark, A. C. Budd, L. M. Alleva, and W. B. Cowden, "Human malarial disease: a consequence of inflammatory cytokine release,” Malaria Journal, vol. 5, p. 85, 2006. 
[80] R. Ralf, "Malarial fever: hemozoin is involved but Toll-free," Proceedings of the National Academy of Sciences of United States of America, vol. 104, no. 6, pp. 1743-1744, 2007.

[81] P. Peggy, N. Fanny, G. Nadege, L. Eicke, G. M. Brian, and A. Vet al, "Malaria hemozoin is immunologically inert but radically enhances innate responses by presenting malaria DNA to Toll-like receptor 9," Proceedings of the National Academy of Sciences of United States of America, vol. 104, no. 6, pp. 1919-1924, 2007.

[82] G. A. Awandare, Y. Ouma, C. Ouma et al., "Role of monocyte-acquired hemozoin in suppression of macrophage migration inhibitory factor in children with severe malarial anemia," Infection and Immunity, vol. 75, no. 1, pp. 201-210, 2007.

[83] A. A. Lamikanra, M. Theron, T. W. Kooij, and D. J. Roberts, "Hemozoin (malarial pigment) directly promotes apoptosis of erythroid precursors," PLoS One, vol. 4, no. 12, Article ID e8446, 2009.

[84] A. R. Mawson, "The pathogenesis of malaria: a new perspective," Pathogens and Global Health, vol. 107, no. 3, pp. 122-129, 2013.

[85] L. Schofield and G. E. Grau, "Immunological processes in malaria pathogenesis," Nature Reviews Immunology, vol. 5, no. 9, pp. 722-735, 2005.

[86] K. Haldar, S. C. Murphy, D. A. Milner, and T. E. Taylor, "Malaria: mechanisms of erythrocytic infection and pathological correlates of severe disease," Annual Review of $\mathrm{Pa}$ thology: Mechanisms of Disease, vol. 2, no. 1, pp. 217-249, 2007.

[87] C. Murray, Diagnosis of Malaria, E. L. Baron and J. Daily, Eds., , UpToDate 19.3 [desktop application], 2009.

[88] WHO, Management of Severe Malaria. A Practical Handbook, WHO, Geneva, Switzerland, 3rd edition, 2012.

[89] B. Nicoletta, S. Roberta, and D. Sarah, "Malaria diagnosis, therapy, vaccines, and vector control," in Human and Mosquito Lysozymes, M. Prato, Ed., Springer, Berlin, Germany, 2015.

[90] FMoH, National Malaria Guidelines, Ministry of Health. Federal Democratic Republic of Ethiopia, Addis Ababa, Ethiopia, 3rd edition, 2012.

[91] R. H. Barker, J. M. Courval, T. Banchongaksorn, D. F. Wirth, K. Rimwungtragoon, and W. Suwonkerd, "A simple method to detect plasmodium falciparum directly from blood samples using the polymerase chain reaction," The American Journal of Tropical Medicine and Hygiene, vol. 46, no. 4, pp. 416-426, 1992.

[92] S. Kumar and K. Renu, "Recently developed new, sensitive, time-effective and cost-effective diagnostic tests of malaria," Proceeding of Indian National Science Academy, vol. 81, no. 2, pp. 479-483, 2015.

[93] J. C. Mouatcho and J. P. D. Goldring, "Malaria rapid diagnostic tests: challenges and prospects," Journal of Medical Microbiology, vol. 62, no. 10, pp. 1491-1505, 2013.

[94] B.-K. Na, J.-W. Park, H.-W. Lee et al., "Characterization of Plasmodium vivax heat shock protein 70 and evaluation of its value for serodiagnosis of tertian malaria," Clinical and Vaccine Immunology, vol. 14, no. 3, pp. 320-322, 2007.

[95] J. H. Kattenberg, I. Versteeg, S. J. Migchelsen et al., "New developments in malaria diagnostics," Mabs, vol. 4, no. 1, pp. 120-126, 2012.

[96] M. P. G. Jepsen, P. S. Jogdand, S. K. Singh et al., "The malaria vaccine candidate GMZ2 elicits functional antibodies in individuals from malaria endemic and non-endemic areas,"
The Journal of Infectious Diseases, vol. 208, no. 3, pp. 479488, 2013.

[97] S. J. Higgins, K. Xing, H. Kim et al., "Systemic release of high mobility group box 1 (HMGB1) protein is associated with severe and fatal Plasmodium falciparum malaria," Malaria Journal, vol. 12, no. 1, p. 105, 2013.

[98] W. Shawna, "Children with malaria smell more attractive to mosquitoes," The Scientist, 2018.

[99] FMoH, National Strategic Plan for Malaria Prevention, Control and Elimination in Ethiopia 2014-2020, Federal Ministry of Health, Addis Abba, Ethiopia, 2014.

[100] WHO, World Malaria Report, WHO, Geneva, Switzerland, 2013.

[101] G. C. Müller, J. C. Beier, S. F. Traore et al., "Successful field trial of attractive toxic sugar bait (ATSB) plant- spraying methods against malaria vectors in the Anopheles gambiae complex in Mali, West Africa," Malaria Journal, vol. 9, p. $210,2010$.

[102] J. C. Beier, G. C. Muller, W. GU, K. L. Arheart, and Y. Schlein, "Attractive toxic sugar bait (ATSB) methods decimate populations of Anopheles malaria vectors in arid environments regardless of the local availability of favoured sugar-source blossoms," Malaria Journal, vol. 11, p. 31, 2012.

[103] S. P. Sawadogo, A. Niang, E. Bilgo et al., "Targeting male mosquito swarms to control malaria vector density," PLoS One, vol. 12, no. 3, Article ID e0173273, 2017.

[104] P. Kajfasz, "Malaria prevention," International Maritime Health, vol. 60, no. 60, pp. 67-70, 2009.

[105] MAL WEST, Integrated Survillance and Control Programme for West Line Virus and Malaria in Greece, ISGlobal, Barcelona, Spain, 2016.

[106] K. Jaleta, S. Hill, G. Birgersson, H. Tekie, and R. Ignell, "Chicken volatiles repel host-seeking malaria mosquitoes," Malaria Journal, vol. 15, p. 354, 2016.

[107] P. Barreaux, A. M. G. Barreaux, E. D. Sternberg et al., "Priorities for broadening the malaria vector control tool kit," Trends in Parasitology, vol. 33, no. 10, pp. 763-774, 2017.

[108] M. Rowland, N. Durrani, M. Kenward, N. Mohammed, H. Urahman, and S. Hewitt, "Control of malaria in Pakistan by applying deltamethrin insecticide to cattle: a communityrandomised trial," The Lancet, vol. 357, no. 9271, pp. $1837-1841,2001$

[109] A. M. Mahande, F. W. Mosha, J. M. Mahande, and E. J. Kweka, "Role of cattle treated with deltamethrine in areas with a high population of Anopheles arabiensis in Moshi, Northern Tanzania," Malaria Journal, vol. 6, no. 1, p. 109, 2007.

[110] S. Naz, A. Maqbool, M. Ahmad, A. Anjum, and S. Zaman, "Efficacy of ivermectin for control of zoophilic malaria vectors in Pakistan," Pakistan Journal of Zoology, vol. 45, pp. 1585-1591, 2013.

[111] C. J. Chaccour, N. R. Rabinovich, H. Slater et al., "Establishment of the ivermectin research for malaria elimination network: updating the research agenda," Malaria Journal, vol. 14, p. 243, 2015.

[112] L. M. Childs, F. Y. Cai, E. G. Kakani et al., "Disrupting mosquito reproduction and parasite development for malaria control," PLoS Pathogens, vol. 12, no. 12, Article ID e1006060, 2016.

[113] E. Taylor and M. John, "Malaria control with transgenic mosquitoes. Research in translation," PLoS Medicine, vol. 6, no. 2, Article ID e1000020, 2009.

[114] V. Kokoza, A. Ahmed, S. Woon Shin, N. Okafor, Z. Zou, and A. S. Raikhel, "Blocking of Plasmodium transmission by 
cooperative action of Cecropin A and Defensin A in transgenic Aedes aegypti mosquitoes," Proceedings of the National Academy of Sciences, vol. 107, no. 18, pp. 8111-8116, 2010.

[115] I. Hurwitz, A. Fieck, A. Read et al., "Paratransgenic control of vector borne diseases," International Journal of Biological Sciences, vol. 7, no. 9, pp. 1334-1344, 2011.

[116] T. Walker and L. A. Moreira, "Can Wolbachia be used to control malaria?," Memórias Do Instituto Oswaldo Cruz, vol. 106, no. 1, pp. 212-217, 2011.

[117] F. Catteruccia, A. Crisanti, and E. A. Wimmer, "Transgenic technologies to induce sterility," Malaria Journal, vol. 8, no. 2, p. S7, 2009.

[118] D. P. Kwiatkowski, "How malaria has affected the human genome and what human genetics can teach us about malaria," The American Journal of Human Genetics, vol. 77, no. 2, pp. 171-192, 2005.

[119] J. P. Daily and P. Sabeti, "A malaria fingerprint in the human genome?," New England Journal of Medicine, vol. 358, no. 17, pp. 1855-1856, 2008.

[120] P. W. Hedrick, "Population genetics of malaria resistance in humans," Heredity, vol. 107, no. 4, pp. 283-304, 2011.

[121] M. M. Meremikwu, S. Donegan, D. Sinclair, E. Esu, and C. Oringanje, "Intermittent preventive treatment for malaria in children living in areas with seasonal transmission," Cochrane Database of Systematic Reviews, vol. 2, no. 2, p. CD003756, 2012.

[122] H. Alout, B. Krajacich, J. Meyers et al., "Evaluation of ivermectin mass drug administration for malaria transmission control across different West African environments," Malaria Journal, vol. 13, p. 417, 2014.

[123] F. Jacquerioz and A. Croft, "Drugs for preventing malaria in travelers," Cochrane Database of Systematic Reviews, no. 4, Article ID CD006491, 2009.

[124] N. M. Mbeye, F. O. ter Kuile, M.-A. Davies et al., "Cotrimoxazole prophylactic treatment prevents malaria in children in sub-Saharan Africa: systematic review and metaanalysis," Tropical Medicine \& International Health, vol. 19, no. 9, pp. 1057-1067, 2014.

[125] B. Coulibaly, M. Pritsch, M. Bountogo et al., "Efficacy and safety of triple combination therapy with artesunate-amodiaquine-methylene blue for falciparum malaria in children: a randomized controlled trial in Burkina Faso," The Journal of Infectious Diseases, vol. 211, no. 5, pp. 689-697, 2015.

[126] M. Sulyok, T. Rückle, A. Roth et al., "DSM265 for Plasmodium falciparum chemoprophylaxis: a randomised, double blinded, phase 1 trial with controlled human malaria infection," The Lancet Infectious Diseases, vol. 17, no. 6, pp. 636-644, 2017.

[127] J. A. Thomas, M. S. Y. Tan, C. Bisson et al., “A protease cascade regulates release of the human malaria parasite Plasmodium falciparum from host red blood cells," Nature Microbiology, vol. 3, no. 4, pp. 447-455, 2018.

[128] B. Greenwood, "Malaria vaccines," Acta Tropica, vol. 95, no. 3, pp. 298-304, 2005.

[129] H. Janet, S. Rima, N Timothy et al., "Tools and strategies for malaria control and elimination: what do we need to achieve a grand convergence in malaria?," PLoS Biology, vol. 14, no. 3, Article ID e1002380, 2016.

[130] H. Camila, Y. Justin, Z. Irfan, and E. Patrick, “Advances in malaria vaccine development: report from the 2017 malaria vaccine symposium," Meeting Report, vol. 2, p. 34, 2017.

[131] J. W. Bennett, A. Yadava, D. Tosh, J. Sattabongkot, J. Komisar, and L. A. Ware, "Phase 1/2a trial of plasmodium vivax malaria vaccine candidate vmp001/AS01B in malarianaïve adults: adults: safety, immunogenicity, and efficacy," PLoS Neglected Tropical Disease, vol. 10, no. 2, Article ID e0004423, 2016.

[132] S. J. Draper, B. K. Sack, C. R. King et al., "Malaria vaccines: recent advances and new horizons," Cell Host \& Microbe, vol. 24, no. 1, pp. 43-56, 2018.

[133] C. Arama and M. Troye-Blomberg, "The path of malaria vaccine development: challenges and perspectives," Journal of Internal Medicine, vol. 275, no. 5, pp. 456-466, 2014.

[134] Y.-K. Goo, E.-J. Seo, Y.-k. Choi et al., "First characterization of Plasmodium vivax liver stage antigen (PvLSA) using synthetic peptides," Parasites \& Vectors, vol. 7, no. 1, p. 64, 2014.

[135] R. J. Longley, A. M. Salman, M. G. Cottingham et al., "Comparative assessment of vaccine vectors encoding ten malaria antigens identifies two protective liver-stage candidates,” Scientific Reports, vol. 5, p. 11820, 2015.

[136] I. Sagara, A. Dicko, R. D. Ellis et al., "A randomized controlled phase 2 trial of the blood stage AMA1-C1/Alhydrogel malaria vaccine in children in Mali," Vaccine, vol. 27, no. 23, pp. 3090-3098, 2009.

[137] H. M. El Sahly, S. M. Patel, R. L. Atmar et al., "Safety and immunogenicity of a recombinant nonglycosylated erythrocyte binding antigen 175 Region II malaria vaccine in healthy adults living in an area where malaria is not endemic," Clinical and Vaccine Immunology, vol. 17, no. 10, pp. 1552-1559, 2010.

[138] B. R. Ogutu, O. J. Apollo, D. McKinney et al., "Blood stage malaria vaccine eliciting high antigen-specific antibody concentrations confers no protection to young children in Western Kenya," PloS One, vol. 4, no. 3, Article ID e4708, 2009.

[139] V. S. Chauhan, S. S. Yazdani, and D. Gaur, "Malaria vaccine development based on merozoite surface proteins of Plasmodium falciparum," Human Vaccines, vol. 6, no. 9, pp. 757-762, 2010.

[140] B. Genton, I. Betuela, I. Felger et al., "A recombinant bloodstage malaria vaccine reduces Plasmodium falciparum density and exerts selective pressure on parasite populations in a phase 1-2b trial in Papua New Guinea," The Journal of Infectious Diseases, vol. 185, no. 6, pp. 820-827, 2002.

[141] N. M. Palacpac, E. Ntege, A. Yeka et al., "Phase 1b randomized trial and follow-up study in Uganda of the bloodstage malaria vaccine candidate BK-SE36," PLoS One, vol. 8, no. 5, Article ID e64073, 2013.

[142] S. L. Takala, D. Coulibaly, M. A. Thera et al., "Extreme polymorphism in a vaccine antigen and risk of clinical malaria: implications for vaccine development," Science Translational Medicine, vol. 1, no. 2, p. 2ra5, 2009.

[143] R. D. Ellis, L. B. Martin, D. Shaffer et al., "Phase 1 trial of the Plasmodium falciparum blood stage vaccine MSP1 (42)-C1/ Alhydrogel with and without CPG 7909 in malaria naïve adults," PLoS One, vol. 5, no. 1, Article ID e8787, 2010.

[144] A. V. S. Hill, A. Reyes-Sandoval, G. O'Hara et al., "Primeboost vectored malaria vaccines: progress and prospects," Human Vaccines, vol. 6, no. 1, pp. 78-83, 2010.

[145] E. Malkin, J. Hu, Z. Li et al., "A phase 1 trial of PfCP2.9: an AMA1/MSP1 chimeric recombinant protein vaccine for Plasmodium falciparum malaria," Vaccine, vol. 26, no. 52, pp. 6864-6873, 2008.

[146] P. Hermand, L. Cicéron, C. Pionneau, C. Vaquero, C. Combadière, and P. Deterre, "Plasmodium falciparum proteins involved in cytoadherence of infected erythrocytes 
to chemokine CX3CL1," Scientific Reports, vol. 6, p. 33786, 2016.

[147] L. Y. Bustamante, S. J. Bartholdson, C. Crosnier et al., "A fulllength recombinant Plasmodium falciparum PfRH5 protein induces inhibitory antibodies that are effective across common PfRH5 genetic variants," Vaccine, vol. 31, no. 2, pp. 373-379, 2013.

[148] D. Ito, T. Hasegawa, K. Miura et al., "RALP1 is a rhoptry neck erythrocyte-binding protein of plasmodium falciparum merozoites and a potential blood-stage vaccine candidate antigen," Infection and Immunity, vol. 81, no. 11, pp. 4290-4298, 2013.

[149] Institut Pasteur, Malaria: Plasmodium Falciparum Protein Msp4 Is A Potential Target for A Vaccine, Institut Pasteur, Paris, France, 2018.

[150] C. Pehrson, A. Salanti, T. G. Theander, and M. A. Nielsen, "Pre-clinical and clinical development of the first placental malaria vaccine," Expert Review of Vaccines, vol. 16, no. 6, pp. 613-624, 2017.

[151] C. J. Sutherland, "Surface antigens of Plasmodium falciparum gametocytes-A new class of transmission-blocking vaccine targets?," Molecular and Biochemical Parasitology, vol. 166, no. 2, pp. 93-98, 2009.

[152] M. C. Kapulu, D. F. Da, K. Miura et al., "Comparative assessment of transmission-blocking vaccine candidates against Plasmodium falciparum," Scientific Reports, vol. 5, p. 11193, 2015.

[153] T. Arakawa, A. Komesu, H. Otsuki et al., "Nasal immunization with a malaria transmission-blocking vaccine candidate, Pfs 25 , induces complete protective immunity in mice against field isolates of Plasmodium falciparum," Infection and Immunity, vol. 73, no. 11, pp. 7375-7380, 2005.

[154] H. Hisaeda, A. W. Stowers, T. Tsuboi et al., "Antibodies to malaria vaccine candidates Pvs25 and Pvs28 completely block the ability of Plasmodium vivax to infect mosquitoes," Infection and Immunity, vol. 68, no. 12, pp. 6618-6623, 2000.

[155] A. Molina-Cruz, L. S. Garver, A. Alabaster et al., "The human malaria parasite Pfs 47 gene mediates evasion of the mosquito immune system," Science, vol. 340, no. 6135, pp. 984-987, 2013.

[156] F. Angrisano, K. A. Sala, D. F. Da et al., "Targeting the conserved fusion loop of HAP2 inhibits the transmission of Plasmodium berghei and falciparum," Cell Reports, vol. 21, no. 10, pp. 2868-2878, 2017.

[157] D. Nega, A. Alemu, and G. Tasew, "Malaria vaccine development: recent advances alongside the barriers," Journal of Bacteriology and Parasitology, vol. 7, no. 6, p. 300, 2016.

[158] WHO, WHO Traditional Medicine Strategy: 2014-2023, WHO, Geneva, Switzerland, 2013.

[159] A. M. Agbor, S. Naidoo, and A. M. Mbia, "The role of traditional healers in tooth extractions in Lekie Division, Cameroon," Journal of Ethnobiology and Ethnomedicine, vol. 7, no. 1, p. 15, 2011

[160] A. Asmare and N. B. Kesara, "A review of ethnopharmacology of the commonly used antimalarial herbal agents for traditional medicine practice in Ethiopia," African Journal of Pharmacy and Pharmacology, vol. 9, no. 25, pp. 615-627, 2015.

[161] P. Rasoanaivo, C. W. Wright, M. L. Willcox, and B. Gilbert, "Whole plant extracts versus single compounds for the treatment of malaria: synergy and positive interactions," Malaria Journal, vol. 10, no. S1, p. S4, 2011.

[162] A. C. C. Aguiar, E. M. d. Rocha, N. B. d. Souza, T. C. França, and A. U. Krettli, "New approaches in antimalarial drug discovery and development: a review," Memórias Do Instituto Oswaldo Cruz, vol. 107, no. 7, pp. 831-845, 2012.

[163] T. Deressa, Y. Mekonnen, and A. Animut, "In Vivo antimalarial activities of Clerodendrum myricoides, Dodonea angustifolia and Aloe debrana against Plasmodium berghei," Ethiopian Journal of Health Development, vol. 24, no. 1, pp. 25-29, 2010.

[164] G. Mequanint, "Antimalarial activity of methanolic extract of Phytolacca dodecandra leaves against Plasmodium berghei infected Swiss albino mice," International Journal of Pharmacology and Clinical Sciences, vol. 3, no. 3, pp. 39-45, 2014.

[165] J. Abdela, E. Ephrem, and S. Workineh, "In Vivo antimalarial activity of solvent fractions of the leaves of Justicia schimperiana Hochst. Ex nees against Plasmodium berghei in mice," Ethiopian Pharmaceutical Journal, vol. 30, pp. 95-108, 2014.

[166] M. Adugna, F. Teka, T. Wintana, and P. Admasu, "In vivo antimalarial activity of crude extract of aerial part of Artemisia abyssinica against Plasmodium berghei in mice," Global Journal of Pharmacology, vol. 8, no. 3, pp. 460-468, 2014.

[167] T. Bihonegn, Study on the in Vivo Antimalarial Activity of Solvent Fractions of the Leaf of Vernonia Amygdalina (Asteraceae) against Plasmodium berghei in Mice [dissertation]. Ethiopia, Addis Ababa University, Addis Ababa, Ethiopia, 2016.

[168] N. Mohammed, M. Abdulwuhab, and F. Mohammed, "Antimalarial activity of crude extract of Buddleja Polystachya fresen (buddlejacea) against Plasmodium berghei in mice," IOSR Journal of Pharmacy and Biological Sciences, vol. 11, no. 05, pp. 27-35, 2016.

[169] S. Fentahun, E. Makonnen, T. Awas, and G. Mirutse, "In vivo antimalarial activity of crude extracts and solvent fractions of leaves of Strychnos mitis in Plasmodium berghei infected mice," BMC Complementary and Alternative Medicine, vol. 17, p. 13, 2017.

[170] A. Toma, S. Deyno, S. Eyado, and A. Fikru, "In vivo antimalarial activity of solvent fractions of Echinops kebericho roots against Plasmodium berghei infected mice," EC Microbiology, vol. 12, no. 5, pp. 204-212, 2017.

[171] N. Alelign, M. Giday, T. Teklehaymanot, and A. Animut, "Ethnobotanical survey of antimalarial plants in AwashFentale District of Afar Region of Ethiopia and in vivo evaluation of selected ones against Plasmodium berghei," Asian Pacific Journal of Tropical Biomedicine, vol. 8, no. 1, pp. 73-78, 2018.

[172] E. M. Obasa, B. G. Alemu, S. T Berkessa et al., "Antimalarial activity of selected Ethiopian medicinal plants in mice," Journal of Pharmacy and Pharmacognosy Research, vol. 6, no. 1, pp. 57-64, 2018.

[173] D. Nureye, S. Assefa, T. Nedi, and E. Engidawork, "In vivo antimalarial activity of the $80 \%$ methanolic root bark extract and solvent fractions of Gardenia ternifolia Schumach. \& Thonn. (Rubiaceae) against Plasmodium berghei," EvidenceBased Complementary and Alternative Medicine, vol. 2018, Article ID 9217835, 10 pages, 2018.

[174] K. Na-Bangchang and J. Karbwang, "Current status of malaria chemotherapy and the role of pharmacology in antimalarial drug research and development," Fundamental \& Clinical Pharmacology, vol. 23, no. 4, pp. 387-409, 2009.

[175] P. G. Bray, R. E. Martin, L. Tilley, S. A. Ward, K. Kirk, and D. A. Fidock, "Defining the role of PfCRT in Plasmodium 
falciparum chloroquine resistance," Molecular Microbiology, vol. 56, no. 2, pp. 323-333, 2005.

[176] WHO, Guidelines for the Treatment of Malaria, WHO, Geneva, Switzerland, 2010.

[177] K. Raynes, "Invited review Bisquinoline antimalarials: their role in malaria chemotherapy," International Journal for Parasitology, vol. 29, no. 3, pp. 367-379, 1999.

[178] J. Achan, A. Talisuna, A. Erhart et al., "Quinine, an old antimalarial drug in a modern world: role in the treatment of malaria," Malaria Journal, vol. 10, p. 144, 2011.

[179] U. Bronner, P. C. Divis, A. Farnert, and B. Singh, "Swedish traveller with Plasmodium knowlesi malaria after visiting Malaysian Borneo: a case report," Malaria Journal, vol. 8, no. 1, p. $15,2009$.

[180] I. Strivastava and A. Vaidya, "A mechanism for the synergistic antimalarial action of atovaquone and proguanil," Antimicrobial Agents and Chemotherapy, vol. 43, no. 6, pp. 1334-1339, 1999.

[181] M. P. Grobusch, A. Egan, R. D. Gosling, and R. D. Newman, "Intermittent preventive therapy for malaria: progress and future directions," Current Opinion in Infectious Diseases, vol. 20, no. 6, pp. 613-620, 2007.

[182] Roche, FANSIDAR Product Information, http://www. medicines.org.au/pdf, 2005.

[183] N. White, S. Pukrittayakamee, T. Hien et al., "Malaria," Lancet, vol. 383, pp. 723-735, 2013.

[184] A. M. Dondorp, S. Yeung, L. White et al., "Artemisinin resistance: current status and scenarios for containment," Nature Reviews Microbiology, vol. 8, no. 4, pp. 272-280, 2010.

[185] CDC, Guidelines for Treatment of Malaria in the United States Pdf, 2013.

[186] G. Reddy, S. Reddy, and S. Reddy, "Development and validation of A stability indicating liquid chromatographic method for simultaneous estimation of Arterolane maleate and piperaquine phosphate in combined dosage form," Oriental Journal of Chemistry, vol. 29, no. 4, pp. 1371-1380, 2013.

[187] R. S. Chandra, J. Orazem, D. Ubben, S. Duparc, J. Robbins, and P. Vandenbroucke, "Creative solutions to extraordinary challenges in clinical trials: methodology of a phase III trial of azithromycin and chloroquine fixed-dose combination in pregnant women in Africa," Malaria Journal, vol. 12, p. 122, 2013.

[188] Bvgh, BIO Ventures for Global Health. Malaria Pipelines, https://bvgh.org/neglected-disease-product-pipelines/ malaria-pipelines/, 2015.

[189] S. Rajapakse, C. Rodrigo, and S. D. Fernando, "Tafenoquine for preventing relapse in people with Plasmodium vivax malaria," Cochrane Database Systematic Reviews, vol. 4, Article ID CD010458, 2015.

[190] A. Marco, W. Jutta, and G. Karine, "Recent advances in malaria drug discovery," Bioorganic \& Medicinal Chemistry Letters, vol. 23, no. 10, pp. 2829-2843, 2013.

[191] R. E. Coleman, A. M. Clavin, and W. K. Milhous, "Gametocytocidal and sporontocidal activity of antimalarials against Plasmodium berghei anaka in icr mice and Anopheles stephensi mosquitoes," The American Journal of Tropical Medicine and Hygiene, vol. 46, no. 2, pp. 169-182, 1992.

[192] Novartis, Backgrounder: Malaria Initiative Pipeline, https:// www.novartis.com, 2014.

[193] E. A. Ashley and A. P. Phyo, "Drugs in development for malaria," Drugs, vol. 78, no. 9, pp. 861-879, 2018.
[194] N. J. White, T. T. Duong, C. Uthaisin et al., "Antimalarial activity of KAF156 in falciparum and vivax malaria," New England Journal of Medicine, vol. 375, no. 12, pp. 1152-1160, 2016.

[195] J. P. Jain, F. J. Leong, L. Chen et al., "Bioavailability of lumefantrine is significantly enhanced with a novel formulation approach, an outcome from a randomized, openlabel pharmacokinetic study in healthy volunteers," Antimicrobial Agents and Chemotherapy, vol. 61, no. 9, pp. e00868-17, 2017.

[196] M. A. Phillips, J. Lotharius, K. Marsh et al., "A long-duration dihydroorotate dehydrogenase inhibitor (DSM265) for prevention and treatment of malaria," Science Translational Medicine, vol. 7, no. 296, p. 296ra111, 2015.

[197] S. Martin, "Antimalarial drugs-what is in use and what is in the pipeline," Archiv der Pharmazie, vol. 341, no. 3, pp. 149-163, 2008.

[198] A. M. Leitgeb, P. Charunwatthana, R. Rueangveerayut et al., "Inhibition of merozoite invasion and transient de-sequestration by sevuparin in humans with Plasmodium falciparum malaria," PLoS One, vol. 12, no. 12, Article ID e0188754, 2017.

[199] K. Chibale, How Africa Is Helping Expand the Global Antimalarial Drug Pipeline, University of Cape, Cape Town, South Africa, Town in Partner with the Conversation Africa, 2016.

[200] S. A. Caldarelli, M. Hamel, J.-F. Duckert et al., "Disulfide prodrugs of albitiazolium (T3/SAR97276): synthesis and biological activities," Journal of Medicinal Chemistry, vol. 55, no. 10, pp. 4619-4628, 2012.

[201] A. Das and M. Dash, "A one-stop novel drug for malaria treatment and control," Journal of Emerging Infectious Disease, vol. 1, no. 2, p. 107, 2016.

[202] ClinicalTrials.gov, First-in-Human Trial of Single Ascending Dose, Multiple Ascending Dose and Malaria Challenge Model in Healthy Subjects NCT03261401, https://clinicaltrials.gov/ ct2/show/study/NCT03261401?view=results, 2017.

[203] Medical Xpress, Promising Malaria Drug to Undergo Clinical Trials, Rutgers University, Camden, NJ, USA, 2016.

[204] ClinicalTrials.gov, First-in-Humans Study of an Oral Plasmodium falciparum Plasma Memberane Protein Inhibitor, St. Jude Children's Research Hospital, West, India, 2016.

[205] J. N. Burrows, S. Duparc, W. E. Gutteridge et al., "New developments in antimalarial target candidate and product profiles," Malaria Journal, vol. 16, no. 1, p. 26, 2017.

[206] S. Fonteilles-Drabek, D. Reddy, and T. N. C. Wells, "Managing intellectual property to develop medicines for the world's poorest," Nature Reviews Drug Discovery, vol. 16, no. 4, pp. 223-224, 2017.

[207] G. W. Birrell, G. D. Heffernan, G. A. Schiehser et al., "Characterization of the preclinical pharmacology of the new 2-aminomethylphenol, JPC-3210, for malaria treatment and prevention," Antimicrobial Agents and Chemotherapy, vol. 62, no. 4, pp. e01335-17, 2018.

[208] S. Pegoraro, M. Duffey, T. D. Otto et al., "SC83288 is a clinical development candidate for the treatment of severe malaria," Nature Communications, vol. 8, p. 14193, 2017.

[209] L. Sandeep and S. Rajasree, "Antimalaria drug development \& pipeline," Journal of Analytical \& Pharmaceutical Research, vol. 5, no. 2, Article ID 00136, 2017.

[210] M. B. Jiménez-Díaz, D. Ebert, Y. Salinas et al., “(+)-SJ733, a clinical candidate for malaria that acts through ATP4 to induce rapid host-mediated clearance ofPlasmodium," 
Proceedings of the National Academy of Sciences, vol. 111, no. 50, pp. E5455-E5462, 2014.

[211] M. Kang, G. Lisk, S. Hollingworth, S. M. Baylor, and S. A. Desai, "Malaria parasites are rapidly killed by dantrolene derivatives specific for the plasmodial surface anion channel," Molecular Pharmacology, vol. 68, no. 1, pp. 34-40, 2005.

[212] G. A. Biagini, N. Fisher, N. Berry et al., "Acridinediones: selective and potent inhibitors of the malaria parasite mitochondrial bc1 complex," Molecular Pharmacology, vol. 73, no. 5, pp. 1347-1355, 2008.

[213] R. M. Beteck, F. J. Smit, R. K. Haynes, and D. D. N'Da, "Recent progress in the development of anti-malarial quinolones," Malaria Journal, vol. 13, no. 1, p. 339, 2014.

[214] D. W. N’Da, C. D. Goodman, B. E. Sleebs et al., "Macrolides rapidly inhibit red blood cell invasion by the human malaria parasite, Plasmodium falciparum," BMC Biology, vol. 13, p. 52, 2015.

[215] S. Dana, D. Prusty, D. Dhayal et al., "Potent antimalarial activity of AcriflavineIn VitroandIn vivo," ACS Chemical Biology, vol. 9, no. 10, pp. 2366-2373, 2014.

[216] S. Lilach, "Parasite' could mean more effective treatment for toxoplasmosis and malaria. an unexpected breakthrough looks promising for finding new drugs to treat two diseases," The Conversation, 2018. 